ARTICLE

\title{
The extracellular chaperone Clusterin enhances Tau aggregate seeding in a cellular model
}

Patricia Yuste-Checa (1) 1,2, Victoria A. Trinkaus ${ }^{1,2,3}$, Irene Riera-Tur (1D 4,5, Rahmi Imamoglu (D) 1, Theresa F. Schaller (1) 1,6, Huping Wang (1) 1, Irina Dudanova (1) 4,5, Mark S. Hipp (1) 1,7,8, Andreas Bracher (1) ${ }^{1} \&$ F. Ulrich Hartl 1,2,3凶

Spreading of aggregate pathology across brain regions acts as a driver of disease progression in Tau-related neurodegeneration, including Alzheimer's disease (AD) and frontotemporal dementia. Aggregate seeds released from affected cells are internalized by naïve cells and induce the prion-like templating of soluble Tau into neurotoxic aggregates. Here we show in a cellular model system and in neurons that Clusterin, an abundant extracellular chaperone, strongly enhances Tau aggregate seeding. Upon interaction with Tau aggregates, Clusterin stabilizes highly potent, soluble seed species. Tau/Clusterin complexes enter recipient cells via endocytosis and compromise the endolysosomal compartment, allowing transfer to the cytosol where they propagate aggregation of endogenous Tau. Thus, upregulation of Clusterin, as observed in AD patients, may enhance Tau seeding and possibly accelerate the spreading of Tau pathology.

\footnotetext{
${ }^{1}$ Department of Cellular Biochemistry, Max Planck Institute of Biochemistry, Martinsried, Germany. ${ }^{2}$ Aligning Science Across Parkinson's (ASAP) Collaborative Research Network, Chevy Chase, MD, USA. ${ }^{3}$ Munich Cluster for Systems Neurology (SyNergy), Munich, Germany. ${ }^{4}$ Department of Molecules -Signaling-Development, Max Planck Institute of Neurobiology, Martinsried, Germany. ${ }^{5}$ Molecular Neurodegeneration Group, Max Planck Institute of Neurobiology, Martinsried, Germany. ${ }^{6}$ Present address: Institute for Molecular Medicine, University Medical Center of the Johannes Gutenberg-University Mainz, Mainz, Germany. ${ }^{7}$ Present address: Department of Biomedical Sciences of Cells and Systems, University Medical Center Groningen, University of Groningen, Groningen, The Netherlands. ${ }^{8}$ Present address: School of Medicine and Health Sciences, Carl von Ossietzky University Oldenburg,

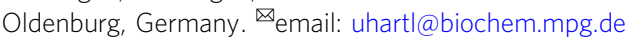


P rogression of several neurodegenerative diseases, prominently including tauopathies such as frontotemporal dementia (FTD) and Alzheimer's disease (AD), is driven by the spreading of aggregate pathology across brain regions in a prion-like seeding mechanism ${ }^{1-5}$. Tau aggregate spreading involves the exposure of aggregate seeds to the extracellular milieu ${ }^{6,7}$, suggesting that extracellular protein quality control factors may modulate disease progression ${ }^{8}$. Clusterin (Clu; apolipoprotein J) is a $\sim 70 \mathrm{kDa}$ glycoprotein with chaperone-like properties found abundantly in plasma and extracellular fluid ${ }^{8-10}$. During its passage through the secretory pathway, immature Clu is extensively $\mathrm{N}$-glycosylated and cleaved into $\alpha$ and $\beta$-chains, which remain linked by disulfide bonds (Supplementary Fig. 1a). Although mainly a secreted chaperone, Clu is also found intracellularly under stress conditions ${ }^{9}$. Clu stabilizes unfolded proteins against aggregation and can inhibit fibril formation of amyloid $\beta(A \beta)$ and other amyloidogenic proteins in vitro, consistent with the function of an ATP-independent "holdase" chaperone ${ }^{10-15}$.

The CLU gene ranks third among genetic risk factors for lateonset $\mathrm{AD}^{16,17}$. However, the mechanism by which Clu modulates $\mathrm{AD}$ pathology remains unclear, as $\mathrm{Clu}$ has been associated with both neuroprotective and neurotoxic effects in $\mathrm{AD}^{15,18-25}$. Clu protein expression is upregulated in $\mathrm{AD}$ patient brain and cerebrospinal fluid ${ }^{26,27}$, localizing with $A \beta$ deposits in senile plaques $^{28-30}$. Evidence has been presented that Clu can mediate uptake of $\mathrm{A} \beta$ by microglia via the endosomal pathway and is involved in clearance of $A \beta$ via the blood-brain barrier ${ }^{31-33}$. On the other hand, enhancement of $\mathrm{A} \beta$ toxicity by Clu has also been reported $^{24,25}$ and elevated plasma levels of Clu were found to be associated with rapid progression of $\mathrm{AD}$, suggesting that $\mathrm{Clu}$ could be a driver of pathology 34,35 . Little is known about the possible role of $\mathrm{Clu}$ in the progression of Tau pathology, which strongly correlates with the severity of $\mathrm{AD}^{36-38}$. Interestingly, Clu was identified as an interactor of soluble Tau in AD brain ${ }^{39}$. More recently, it was shown that $\mathrm{Clu}$ also colocalizes with intracellular Tau aggregates and may provide a protective function by inhibiting fibril formation ${ }^{40}$. Given the predominant role of extracellular $\mathrm{Clu}$ as the chaperone active form, it remains to be understood whether Clu modulates transcellular Tau seeding and influences overall pathology.

Here we analyzed the effect of Clu on the seeding competence of Tau aggregates formed in vitro and in cells. Our results show that $\mathrm{Clu}$ can strongly enhance Tau aggregate propagation by binding and stabilizing seeding active Tau species for cellular uptake. Thus, upregulation of $\mathrm{Clu}$ in $\mathrm{AD}$ has the potential to accelerate disease progression by enhancing the seeding competence of Tau aggregates.

\section{Results}

Clusterin potentiates seeding of Tau aggregates. To test whether Clu interferes with aggregate seeding of Tau, ${ }^{10,14}$, we purified chaperone-active Clu upon recombinant expression in HEK293EBNA cells (Supplementary Fig. 1b, c). We measured aggregate seeding with TauRD-YT cells, a HEK293T cell line stably coexpressing the repeat domain of Tau (TauRD; residues 244-372 with FTD mutations $\mathrm{P} 301 \mathrm{~L} / \mathrm{V} 337 \mathrm{M}$ ) fused to YFP or mTurquoise2, whose co-aggregation during fibril formation results in fluorescence resonance energy transfer $\left(\right.$ FRET) ${ }^{41}$ (Fig. 1a). Seed aggregates were generated with recombinant, cysteine-free TauRD (Tau residues 244-371, C291A/P301L/C322A/V337M) to avoid the use of reducing agents that might interfere with Clu function. Mutation of the two cysteines in TauRD avoids the formation of intramolecular disulfide bonds that slows fibril formation ${ }^{42}$. Lipofectamine was used to render seed uptake independent of cellular machinery for internalization. The TauRD is critical for aggregation and forms the core of Tau fibrils ${ }^{43,44}$.

TauRD rapidly formed thioflavin $\mathrm{T}$ (ThT)-positive fibrillar aggregates in vitro, induced by heparin ${ }^{45}$ (Fig. 1b, Supplementary Fig. 2a). The addition of Clu at an equimolar ratio to TauRD extended the lag phase and slowed fibril elongation ${ }^{46,47}$ but did not prevent fibril formation (Fig. 1b, Supplementary Fig. 2a). To observe seeding, we next transferred small quantities of TauRD ( $0.05 \mathrm{ng}$ to $14 \mathrm{ng}$ with lipofectamine) after different times of in vitro aggregation to TauRD-YT cells, followed by an analysis of endogenous aggregate formation by flow cytometry of FRET positive cells and fluorescence microscopy (Fig. 1a, c, d, Supplementary Fig. 2b). Seeding competent TauRD accumulated with kinetics similar to the formation of ThT-positive aggregates (Fig. 1b, c). The presence of $\mathrm{Clu}$ in the aggregation reaction delayed the appearance of seed material (0\% FRET positive cells after $1 \mathrm{~h}$ aggregation time, Fig. 1c, d). However, once ThT-positive aggregates formed (from $2 \mathrm{~h}$ on, Fig. 1b), the Clu-containing aggregation reaction surprisingly presented a markedly increased seeding potency resulting in $\sim 80 \%$ FRETpositive cells (exceeding the linear range of the assay) compared to $\sim 30 \%$ FRET-positive cells with TauRD aggregates alone (Fig. 1c, d, Supplementary Fig. 2b). When aggregation reactions were diluted 280 -fold, seeding without Clu was virtually abolished, but was still measurable in the presence of $\mathrm{Clu}$, resulting in $\sim 25 \%$ of aggregate containing cells (Fig. 1c). Again, the kinetics of seed formation correlated with the delayed formation of ThT-positive aggregates (Fig. 1b, c). The FRETpositive inclusions formed in cells with and without $\mathrm{Clu}$ were morphologically similar and stained with the amyloid dye X34 (Fig. 1d, Supplementary Fig. 2c). Clu alone neither formed ThTpositive species nor induced Tau aggregation when added to cells (Supplementary Fig. 2d). Titration experiments using seed material from the plateau phase of aggregation (Fig. 1b) showed that Clu increased seeding potency $\sim 25$-fold (defined as \% FRETpositive cells/ng TauRD) (Fig. 1e, Supplementary Fig. 2e). To test the effect of Clu on seeding in cells with the unperturbed plasma membrane, we omitted the transfection reagent. Under these conditions, Clu still increased the seeding potency of TauRD aggregates approximately eightfold (Supplementary Fig. 3). However, as expected, higher amounts of TauRD and TauRD/ Clu aggregates were necessary to observe aggregate seeding.

The effect of Clu on TauRD aggregation and seeding is concentration-dependent, since increasing the ratio of $\mathrm{Clu}$ relative to TauRD further delayed amyloid formation in vitro and increased seeding potency (Supplementary Fig. 4a, b). However, the effect on seeding potency is saturated at a 1:1 molar ratio of TauRD:Clu (Supplementary Fig. $4 \mathrm{~b}$ ).

As TauRD is a highly charged protein (21 positively and 10 negatively charged amino acids), we tested whether the effect of Clu on TauRD seeding is dependent on electrostatic interactions. TauRD and TauRD/Clu aggregates (Fig. 1a) were incubated with PBS or high salt buffer (PBS/500 $\mathrm{mM} \mathrm{NaCl}$ ) prior to addition to TauRD-YT cells. Incubation with high salt buffer resulted in a general increase in seeding (Supplementary Fig. 4c), suggesting that high ionic strength may stabilize seeding competent TauRD species. Importantly, Clu increased seeding potency both in PBS and in the high salt buffer (Supplementary Fig. 4c), consistent with hydrophobic forces playing a role in the Clu-TauRD interaction.

In order to test the effect of Clu on preformed Tau aggregates, Clu was added to the aggregation reaction once the ThT plateau was reached (at $1 \mathrm{~h}$ or $24 \mathrm{~h}$ after initiating aggregation, Supplementary Fig. 4d). When added at an equimolar ratio to TauRD aggregates, Clu amplified seeding competence $\sim 3-4$-fold 


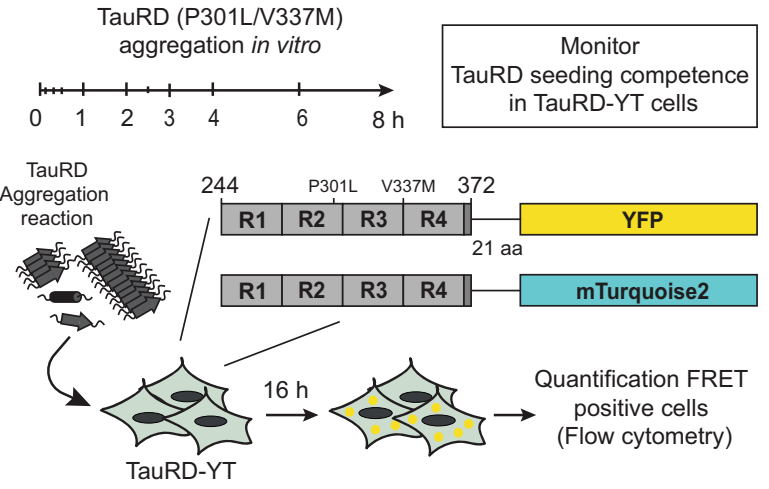

b

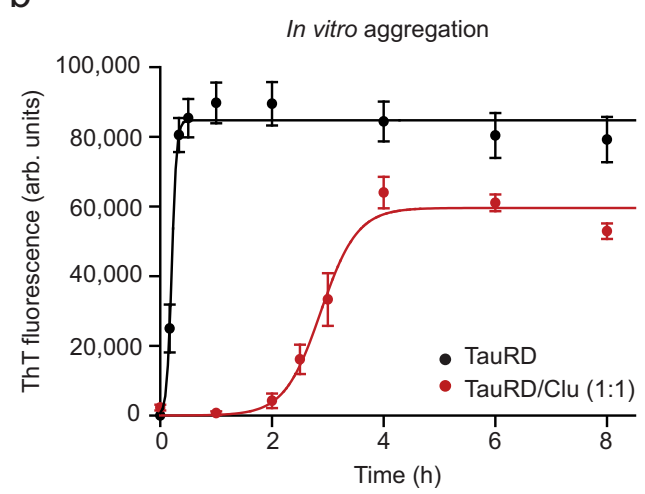

C

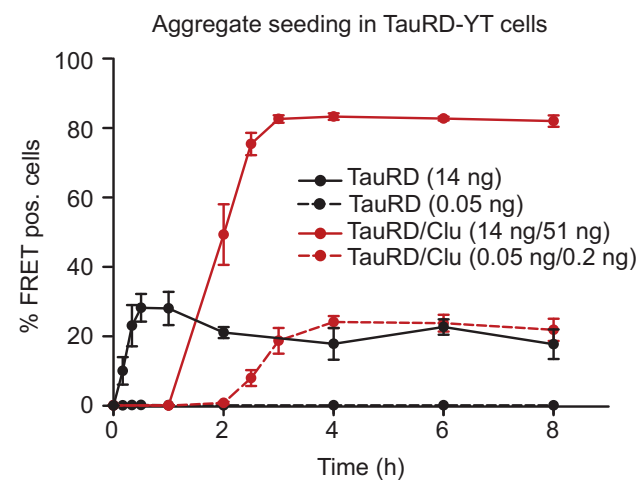

f

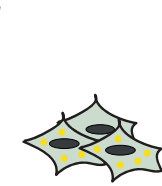

TauRD-YT cells containing aggregates d

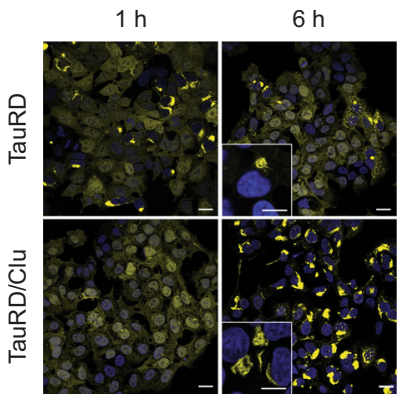

e

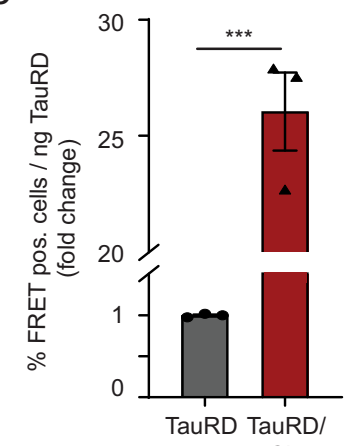

Clu



Fig. 1 Clusterin potentiates seeding of Tau aggregates. a Workflow of the seeding experiment. At the times indicated, samples were removed from TauRD aggregation reactions with or without $\mathrm{Clu}$, and added with or without transfection reagent (lipofectamine) to reporter cells co-expressing TauRD fused to the FRET pair of YFP and mTurquoise2 (TauRD-YT). The fraction of cells containing FRET-positive (pos.) aggregates was quantified by flow cytometry. $\mathbf{b}$ TauRD amyloid formation in aggregation reactions of $10 \mu \mathrm{M}$ TauRD without (black) or with equimolar Clu (red) as monitored by ThT fluorescence. Averages \pm SEM ( $n=10$ independent experiments). arb.units, arbitrary units. c Effect of Clu on the formation of seeds that induce aggregation of endogenous TauRD in cells. Seed formation was analyzed as described in (a). Reporter cells were transfected with aggregation reactions containing $14 \mathrm{ng}$ (solid lines) or $0.05 \mathrm{ng}$ (dashed lines) TauRD and $51 \mathrm{ng}$ (solid lines) or $0.2 \mathrm{ng}$ (dashed lines) Clu, respectively (molar ratio Clu:TauRD 1:1). Averages \pm SEM ( $n=3$ independent experiments). d Representative fluorescence microscopy images of TauRD-YT cells seeded with TauRD (14 ng TauRD) from the plateau phase of aggregation (TauRD, $1 \mathrm{~h}$ reaction time; TauRD/Clu, $6 \mathrm{~h}$ reaction time (b)). TauRD-YFP and DAPI nuclear staining signals are shown in yellow and blue, respectively. Scale bars, $20 \mu \mathrm{m}$ for overview panels and $10 \mu \mathrm{m}$ for insets. e Fold change of seeding potency of TauRD aggregation reactions containing Clu (red) compared to control reactions without Clu (gray). Bar graphs represent the average slope \pm SEM ( $n=3$ independent experiments) from the linear regression analyses described in Supplementary Fig. 2e. ${ }^{\star \star \star} p<0.001\left(p=1.2 \times 10^{-4}\right)$ by two-tailed Student's $t$-test. f Effect of Clu on seeding potency of TauRD-YT cell lysates containing TauRD-YT aggregates. Whole-cell lysates of FRET-positive (pos.) TauRD-YT cells were incubated with or without Clu. Fold change of TauRD seeding potency expressed per ng of TauRD-YT in cell lysates upon treatment with increasing Clu (molar ratios TauRD-YT:Clu 1:2, 1:10 and 1:20). Bar graphs represent the average slope $\pm \operatorname{SEM}\left(n=3\right.$ independent experiments) from the linear regression analyses shown in Supplementary Fig. 4 e. ${ }^{\star \star \star} p<0.001$ (-Clu vs. TauRD-YT:Clu 1:10 $p=8 \times 10^{-4}$; - Clu vs. TauRD-YT:Clu 1:20 $p=1.1 \times 10^{-8}$ ) by one-way ANOVA with Bonferroni post hoc test.

(Supplementary Fig. 4d), similar to when present during aggregation (Fig. 1c). Thus, Clu may act on preexistent fibrils or on aggregate species present in equilibrium.

The use of heparin in seed production results in a nonphysiological conformation of Tau fibrils ${ }^{44}$. To exclude that the observed effect on TauRD seeding is dependent on heparin, we, therefore, investigated the effect of Clu on seeding by TauRD aggregates produced in cells. Cells were lysed under mild conditions in the presence of non-ionic detergent $(0.05 \%$ Triton $\mathrm{X}-100)$ without sonication to preserve the structural properties of the aggregates. Lysates from TauRD-YT cells containing aggregates were incubated with increasing concentrations of Clu 
a

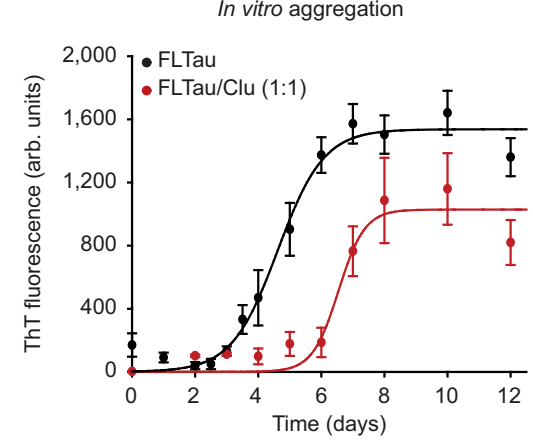

b

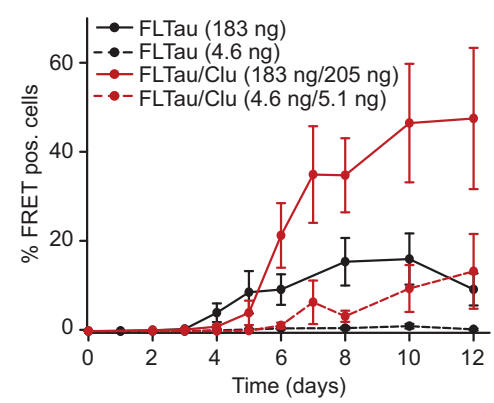

C

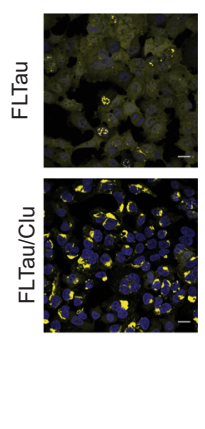

d

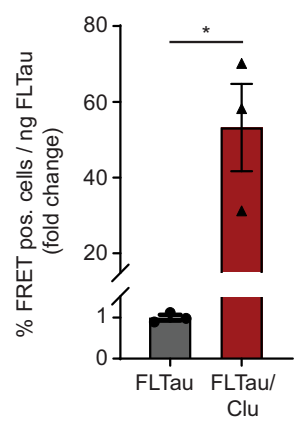

e

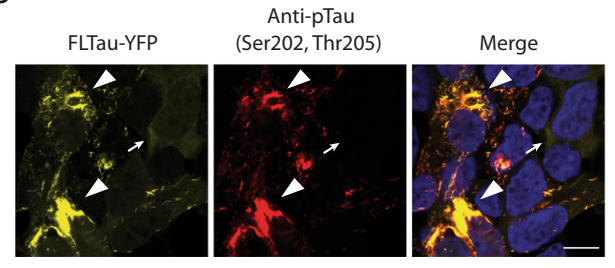

f

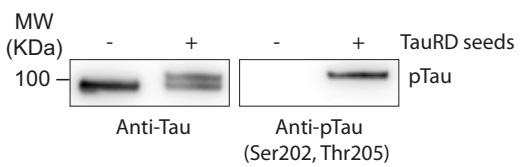

g

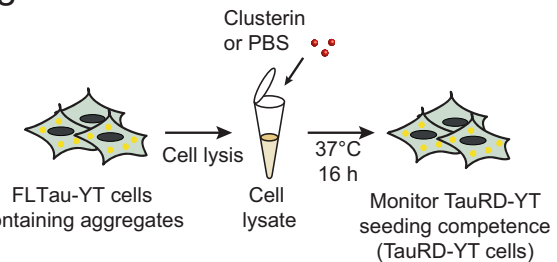

Aggregate seeding in TauRD-YT cells

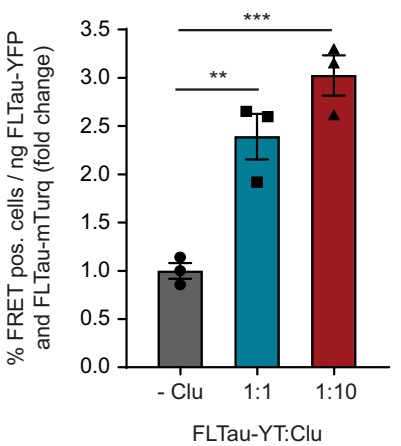

Fig. 2 Clusterin enhances the seeding potency of FLTau aggregates. a Effect of Clu on the kinetics of aggregation of full-length (FL) Tau (10 $\mu M$ ) monitored by ThT fluorescence. FLTau/Clu molar ratio was 1:1. Averages \pm SEM ( $n=5$ independent experiments). arb.units, arbitrary units. b Formation of seeds in FLTau aggregation reactions without (black) or with Clu $(10 \mu \mathrm{M}$, red) as described in (a). Reporter cells were transfected with aggregation reactions containing $183 \mathrm{ng}$ (solid lines) or $4.6 \mathrm{ng}$ (dashed lines) FLTau and $205 \mathrm{ng}$ (solid lines) or $5.1 \mathrm{ng}$ (dashed lines) Clu, respectively (molar ratio Clu: FLTau 1:1). Averages \pm SEM ( $n=3$ independent experiments). c Representative fluorescence microscopy images of TauRD-YT cells seeded with FLTau aggregation reactions (183 ng FLTau) after reaching the plateau of aggregation (10 days, (a)). TauRD-YFP and DAPI nuclear staining signals are shown in yellow and blue, respectively. Scale bars, $20 \mu \mathrm{m}$. d Fold change of seeding potency of FLTau aggregation reactions containing Clu (red) compared to control reactions without Clu (gray). Bar graphs represent the average slope \pm SEM ( $n=3$ independent experiments) from the linear regression analyses described in Supplementary Fig. 5a. ${ }^{*} p<0.05(p=0.0106)$ by two-tailed Student's $t$-test. Lipofectamine was used as a transfection reagent. $\mathbf{e}$, $\mathbf{f}$ Seeded aggregates of FLTau-YT contain phospho-Tau. ( $n=3$ independent experiments) e Fluorescence microscopy images of FLTau-YT cells seeded with TauRD aggregates. FLTau-YFP and immunostaining of phospho-Tau (pTau, AT8 antibody) are shown in yellow and red, respectively. The AT8 antibody recognizes Tau phosphorylation at both serine 202 and threonine 205 and is widely used to detect Tau paired helical fibrils 36,49 . DAPI nuclear staining signal is additionally shown in blue in the merge. Arrowheads indicate aggregates. The small arrow indicates a cell without aggregates. Scale bar, $10 \mu \mathrm{m} . \mathbf{f}$ Representative immunoblot analysis showing Tau (Tau/Repeat Domain antibody) and phospho-Tau (pTau, AT8 antibody) in FLTau-YT cell lysates from cells treated with or without TauRD seeds. Molecular weight (MW) standards are indicated. $\mathbf{g}$ Clu enhances the seeding potency of FLTau aggregates formed in FLTau-YT cells. Whole-cell lysates of FRET-positive (pos.) FLTau-YT cells were incubated without or with Clu (molar ratios FLTau-YT:Clu 1:1 and 1:10). Bar graphs represent the average slope \pm SEM from the linear regression analyses described in Supplementary Fig. 5 e. Data represent the mean \pm SEM $(n=3$ independent experiments). ${ }^{\star \star} p<0.01$ ( - Clu vs. FLTau-YT:Clu 1:1 $p=0.0058$ ); ${ }^{\star \star *} p<0.001$ ( - Clu vs. FLTau-YT:Clu 1:10 $p=7.9 \times 10^{-4}$ ) by one-way ANOVA with Bonferroni post hoc test. Lipofectamine was used as a transfection reagent.

(Fig. 1f). A strong increase in seeding potency (up to $\sim 60$-fold) was observed upon $\mathrm{Clu}$ addition (Fig. If and Supplementary Fig. 4e). In this case, higher amounts of Clu relative to TauRD (up to $\sim 20$-fold excess) were effective, presumably due to lysate proteins competing for Clu binding with the aggregates.

To exclude the possibility that our findings are limited to the isolated repeat domain of Tau, we next performed experiments with full-length Tau (FLTau 2N4R) aggregates as seeds in cells expressing either TauRD or FLTau FRET constructs. As expected, in vitro amyloid formation of FLTau was $\operatorname{slow}^{42}\left(t_{1 / 2} \sim 4.6\right.$ days, Fig. 2a) and was further delayed in the presence of $\mathrm{Clu}\left(t_{1 / 2} \sim\right.$ 6.6 days, Fig. 2a). Clu dramatically enhanced ( $\sim 55$-fold) the potency of FLTau aggregates to seed TauRD-YT aggregates (Fig. 2b-d and Supplementary Fig. 5a). An even greater potentiation of FLTau seeds ( 100-fold) was observed with cells stably co-expressing FLTau (P301L/V337M) fusion proteins with
YFP or mTurquoise2 (FLTau-YT cells), forming FLTau aggregates (Supplementary Fig. 5b-d).

As Tau aggregates in the patient brain typically contain highly phosphorylated $\mathrm{Tau}^{48}$, we also tested the effect of Clu using cell lysates containing phosphorylated FLTau-YT aggregates as seeds $^{36,49}$ (Fig. 2e-g and Supplementary Fig. 5e). Phosphorylated FLTau-YT aggregates were obtained by seeding naïve FLTau-YT cells with TauRD aggregates formed in vitro (Fig. 1b). Phosphorylation of the resulting aggregates was confirmed by the AT8 antibody $^{36,49}$ (Fig. 2e, f). Clu enhanced the seeding competence of these phospho-Tau aggregates up to threefold (Fig. $2 \mathrm{~g}$ and Supplementary Fig. 5e). The lower effect of Clu on the seeding potency of cellular FLTau aggregates compared to TauRD aggregates (Figs. If and 2g) may be due to the "fuzzy coat" around the core of FLTau fibrils ${ }^{50}$, which may limit Clu binding, or to differential posttranslational modifications, including phosphorylation. 
Physical interaction between Clu and phospho-FLTau containing aggregates was confirmed by co-immunoprecipitation using the AT8 antibody (Supplementary Fig. 5f). However, the AT8 antibody also precipitated unmodified, apparently co-aggregated FL-Tau and thus a direct interaction of Clu with phospho-Tau remains to be demonstrated.

In summary, Clu robustly enhances the potency of TauRD and FLTau aggregates to seed aggregation in cells expressing TauRD or FLTau. This effect is independent of whether Clu is present during initial aggregation or added to preformed aggregates produced in vitro or in cells.

Clusterin stabilizes oligomeric Tau seeds. To biochemically characterize the seeding competent TauRD species, we fractionated the in vitro aggregation reaction by centrifugation. In the absence of $\mathrm{Clu}$, aggregated TauRD was apparently insoluble after $1 \mathrm{~h}$ of aggregation (Fig. 3a, upper panel). When increasing amounts of the supernatant fraction $(\times 10, \times 20, \times 30$ the amount loaded in the upper panel) (Fig. 3a, lower panel) were analyzed, we found $\sim 1.5 \%$ of total TauRD to be soluble. The amount of soluble TauRD increased to $\sim 14 \%$ of the total in the presence of equimolar Clu (Fig. 3a, lower panel). Approximately $13 \%$ of Clu was recovered in the pellet fraction (Fig. 3a, upper panel), suggesting a weak association with insoluble fibrils. The seeding competence of the soluble and insoluble TauRD was compared by measuring the fraction of FRET positive cells per ng of TauRD in the seed material as determined by quantitative immunoblotting (Fig. 3a). The soluble fraction of the aggregation reaction had a much higher specific seeding capacity in the cellular assay than the resuspended pellet (Fig. $3 \mathrm{~b}$ ), indicating that soluble TauRD species are more seeding competent ${ }^{51,52}$. Notably, the specific seeding activity of the Clu-containing supernatant was $\sim 40$-fold higher than that in the absence of Clu (Fig. 3b), suggesting that Clu not only increased the amount of soluble TauRD aggregates but also enhanced their intrinsic seeding activity. To test this possibility directly, we added Clu to the seeding-active, soluble fraction of a TauRD aggregation reaction generated in the absence of Clu. This resulted in an approximately fivefold increase in seeding capacity (Fig. 3c). Thus, Clu not only increases the amount of soluble TauRD seeds when present during aggregation but also enhances the intrinsic seeding activity of preexisting, soluble TauRD species.

The addition of Clu $6 \mathrm{~h}$ after initiating TauRD aggregation still produced a small, but detectable amount of soluble TauRD (Supplementary Fig. 6a), in line with enhanced seeding when Clu is added to preformed aggregates (Fig. 1f, Fig. 2g, and Supplementary Fig. 4d). Surprisingly, a small amount of soluble TauRD was also generated when $\mathrm{Clu}$ was added to the resuspended pellet fraction (Supplementary Fig. 6b). In both cases, $\sim 4-5 \%$ of total Clu is associated with the insoluble TauRD aggregates (Supplementary Fig. 6a-c). Thus, Clu binding to preexistent, insoluble TauRD aggregates generates seeding competent, soluble TauRD.

To determine when during the aggregation reaction Clu interacts with TauRD, we utilized dual-color fluorescence crosscorrelation spectroscopy (dcFCCS). To this end, we generated the cysteine mutant I260C in otherwise cysteine-free TauRD and labeled the protein with Alexa Fluor 532 (TauRD-A532). Residue 260 is situated outside the fibril core ${ }^{44,53}$. Both Clu chains were labeled N-terminally with Alexa Fluor 647 (CluA647). Labeled TauRD at $1 \mu \mathrm{M}$ was added to an aggregation reaction of unlabeled TauRD $(9 \mu \mathrm{M})$. Labeling did not substantially affect aggregation kinetics or seeding properties of the resulting aggregates (Supplementary Fig. 7a, b, labeled TauRD; Fig. 1b, c, unlabeled TauRD) and labeled Clu (1.25 $\mu \mathrm{M})$ enhanced seeding to a similar extent as observed with the unlabeled proteins (Supplementary Fig. 7b, labeled proteins; Supplementary Fig. 4b, unlabeled proteins; TauRD:Clu 8:1). A clear fluorescence cross-correlation signal between TauRDA532 and Clu-A647 was only detectable at $1.5-2 \mathrm{~h}$ after initiation of aggregation (Fig. 3d), when ThT-positive and seeding competent TauRD species had formed (Fig. 3e). Apparently, Clu does not bind to monomeric TauRD but interacts with TauRD aggregates. Based on their diffusion time $(\sim 16.5 \pm 1.3 \mathrm{~ms}$; Supplementary Fig. $7 \mathrm{c})$, the TauRD/Clu complexes formed are on average $\sim 5000 \mathrm{kDa}$ in size, equivalent to Clu bound to TauRD fibrils comprising $~ 300$ TauRD units.

To characterize the interaction of Clu with the soluble, highly seeding competent TauRD, we next measured the FCCS signal between Clu-A647 and TauRD-A532 in the soluble fraction of aggregation reactions. Clu did not interact with monomeric TauRD ( 0 h; Fig. 3 f), but strong interaction with soluble TauRD was detected after aggregation had reached the plateau phase $(6 \mathrm{~h}$; Fig. 3f, Supplementary Fig. 7a). The average size of the soluble TauRD/Clu complexes was $\sim 320 \mathrm{kDa}$ (diffusion time $\sim 4.95 \pm$ $0.19 \mathrm{~ms}$; Supplementary Fig. $7 \mathrm{c}$ ), consistent with one or more Clu stabilizing small TauRD oligomers. The interaction between Clu and TauRD is dynamic, as the FCCS signal was rapidly reduced upon the addition of excess unlabeled Clu (Supplementary Fig. 7d, e). Together these data show that Clu binds and stabilizes soluble Tau oligomers, either when present during ongoing aggregation or when added to preexistent aggregates. These soluble species are highly competent for cellular uptake and seeding of endogenous aggregation.

Seeding competent Tau/Clu species enter cells by endocytosis. To test whether cells incorporate TauRD/Clu seeds by endocytosis, as described for Tau alone ${ }^{54-58}$, we incubated TauRD-YT cells during seeding with Bafilomycin, an inhibitor of the lysosomal $\mathrm{H}^{+}$ATPase $^{59}$, or leucyl-leucyl-O-methylester (LLOME), an agent that accumulates in acidic membrane compartments inducing their rupture ${ }^{60}$. A reduced amount of Tau/Clu aggregates compared to TauRD aggregates was applied to the TauRDYT cells in order to obtain comparable seeding efficiencies. Both compounds increased seeding by TauRD and TauRD/Clu aggregates (in the absence of transfection reagent) to a similar extent and in a concentration-dependent manner (Fig. 4a, b). Thus, seed internalization occurs via endocytosis, with a fraction of seed material presumably undergoing lysosomal degradation in the absence of Bafilomycin or LLOME.

Heparan sulfate proteoglycans (HSPGs) are involved in cell surface binding and internalization of Tau seeds ${ }^{61}$, as well as in the clearance of aberrant extracellular proteins mediated by $\mathrm{Clu}^{31}$. To test the role of this internalization mechanism in Tau/Clu seed uptake, TauRD-YT cells were incubated with increasing concentrations of heparin, an HSPG blocker, and treated with Tau aggregates formed in the presence or absence of Clu. In both cases the number of FRET positive cells strongly decreased in a manner dependent on heparin concentration (Fig. 4c), suggesting that TauRD and TauRD/Clu aggregates are internalized via HSPGs. We next investigated whether Clu and TauRD seeds enter cells together. To this end, we incubated HEK293T cells stably expressing TauRD (P301L/V337M)-mTurquoise2 (TauRD$\mathrm{T}$ cells) with fluorescence-labeled soluble TauRD-A532/CluA647 seeds (Supplementary Fig. 7a, b) for $24 \mathrm{~h}$. Clu-A647 and TauRD-A532 entered the cells as a complex (Fig. 4d-g and Supplementary Fig. 8) and in several cases co-localized with CHMP2a (Fig. 4d and Supplementary Fig. 8a), a subunit of the endosomal sorting complexes required for transport (ESCRT) machinery ${ }^{62}$. Some seed material also co-localized with the 

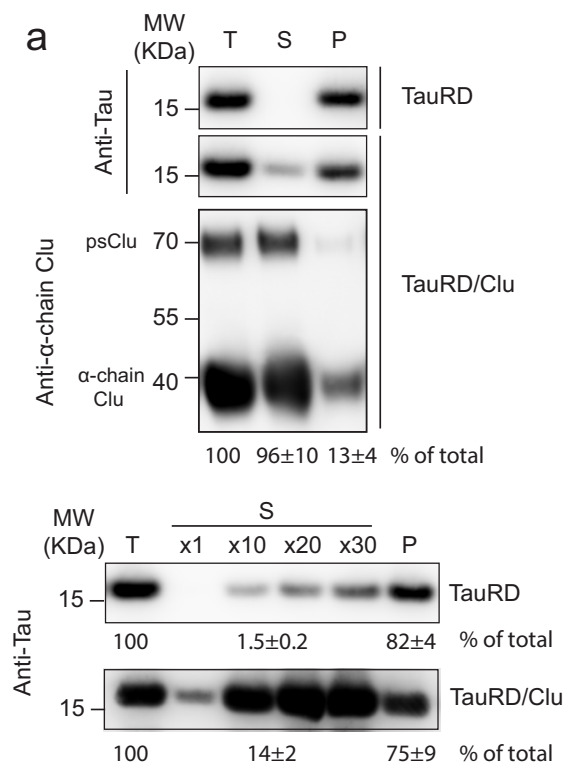

b

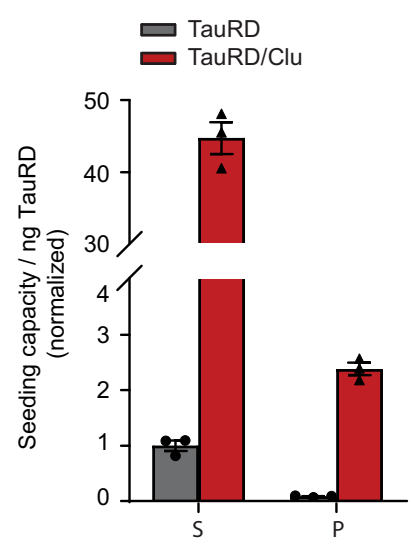

d

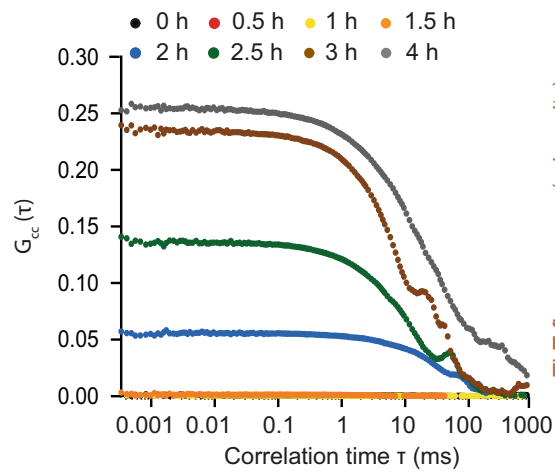

e

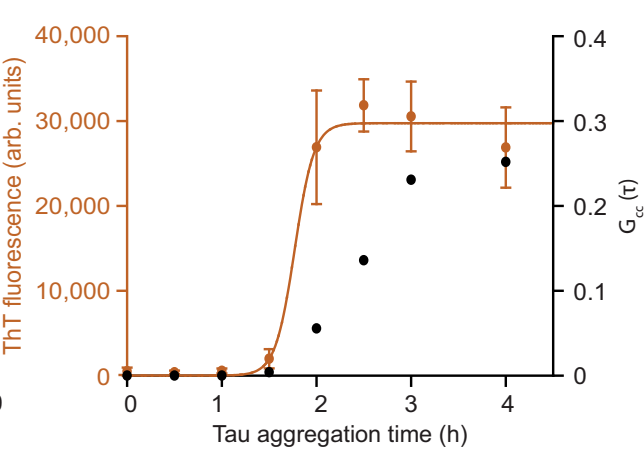

C


f



Fig. 3 Clusterin binds to aggregates and stabilizes oligomeric Tau seeds. a Fractionation by sedimentation of TauRD aggregation reactions without or with Clu (TauRD/Clu). Representative immunoblots of total $(T)$, supernatant (S), and pellet (P) fractions. The small amount of soluble TauRD was visualized and quantified by analyzing increasing amounts of the supernatant fraction (lower panel, $\times 10, \times 20, \times 30$ times the amount loaded in upper panel, $\times 1)$. psClu: pre-secretory Clu. TauRD ( $n=6$ independent experiments) and Clu ( $n=3$ independent experiments) were quantified by densitometry with the amounts in total fractions set to $100 \%$. Averages \pm SEM. Molecular weight (MW) standards are indicated. $\mathbf{b}$ Seeding potency of soluble (S) and pellet (P) fractions of TauRD aggregation reactions formed without (gray) or with Clu (red). Lipofectamine was used as a transfection reagent. Seeding by soluble TauRD without Clu was set to 1 . Averages \pm SEM ( $n=3$ independent experiments). c Effect of Clu on the seeding potency of the soluble fraction of aggregation reactions without Clu. The soluble fraction was incubated for 30 min either with Clu or with PBS prior to addition to cells. Lipofectamine was used as a transfection reagent. Titration of seeding potency was performed as described in Supplementary Fig. 2e. Bar graphs represent the average slope \pm SEM from the linear regression analyses $\left(n=4\right.$ independent experiments). ${ }^{\star \star} p<0.01(p=0.0013)$ by two-tailed Student's $t$-test. $\mathbf{d}$, e Dual-color fluorescence cross-correlation spectroscopy (dcFCCS) analysis of the interaction of TauRD with Clu during aggregation. Aggregation reactions contained 1 $\mu \mathrm{M}$ Alexa Fluor 532 labeled TauRD (TauRD-A532) and $9 \mu \mathrm{M}$ unlabeled TauRD in the presence of Alexa Fluor 647 labeled Clu (Clu-A647; $1.25 \mu \mathrm{M}$ ). Representative experiments are shown ( $n=4$ independent experiments). e Kinetic development of FCCS signal in (d) (right $y$-axis; black) relative to the formation of ThT positive aggregates (left $y$-axis; orange, Supplementary Fig. 7a, data represent the mean \pm SEM ( $n=3$ independent experiments)). arb. units, arbitrary units. $\mathbf{f}$ Clu interacts with soluble oligomeric TauRD species. dcFCCS analysis of TauRD-A532 and Clu-A647 interaction in the soluble fraction of the aggregation reaction immediately upon initiation of aggregation ( $\mathrm{h}$, black) and after reaching the plateau of ThT positive aggregate formation ( 6 h, red, Supplementary Fig. 7a). Representative data are shown ( $n=3$ independent experiments).

danger receptor galectine-8 (GAL-8) (Fig. 4e and Supplementary Fig. 8b), a marker of ruptured endomembranes ${ }^{63}$. After incubation for a further $24 \mathrm{~h}$, we observed incorporation of TauRD-A532 and Clu-A647 into aggregates formed by endogenous TauRD-mTurquoise2 (TauRD-mTurq) (Fig. 4f, g and Supplementary Fig. 8c-f). The level of colocalization of the three fluorophores in seeded TauRD-mTurq aggregates was quantified by plotting their relative intensity profile, extracted from lines manually drawn in mid focal planes (Fig. $4 \mathrm{~g}$ and Supplementary Fig. 8d, f). Co-localization of Tau-A532/Clu-A647 seeds with
CHMP2a, Gal-8, and endogenous aggregates was not detected frequently enough to be reliably quantified.

Thus, following uptake by endocytosis, TauRD/Clu seeds presumably induce vesicle damage ${ }^{54,58}$, allowing their escape into the cytosol where they template aggregation of endogenous TauRD.

Clusterin interferes with seeding of a-Synuclein aggregates. To test whether the effect of Clu on aggregate seeding is Tau specific, we next performed experiments with $\alpha$-Synuclein ( $\alpha$-Syn), which 
a



b

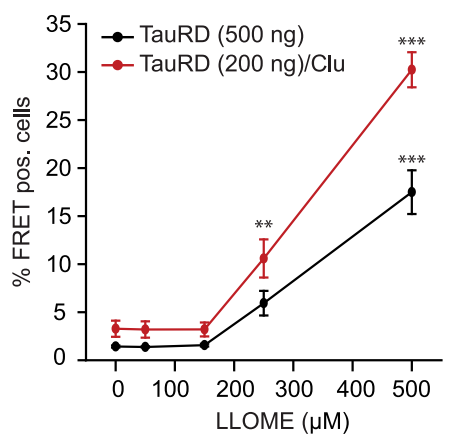

C

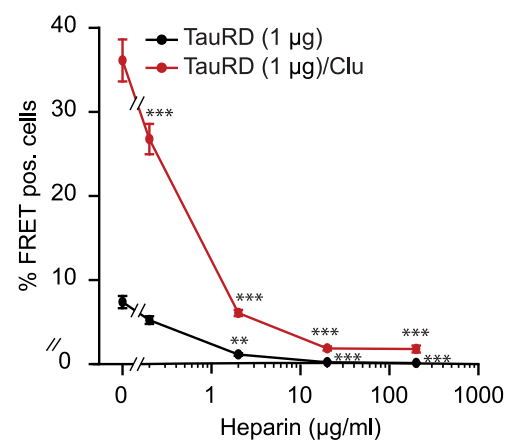

d

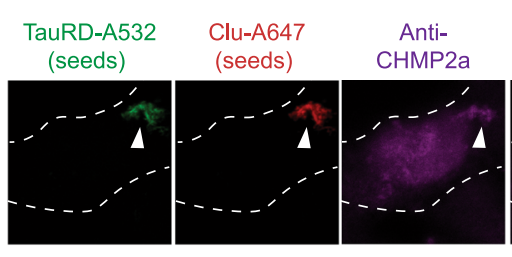

e

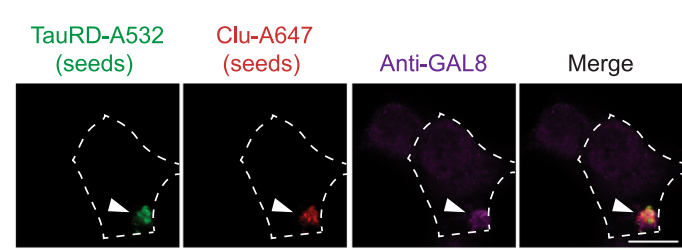

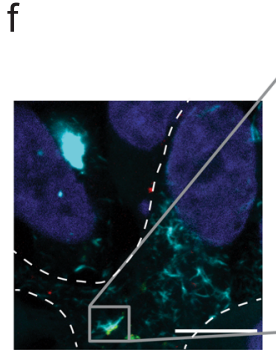

TauRD-mTurq (cell)

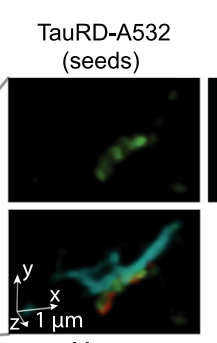

Merge



Fig. 4 Uptake of Clusterin-associated, seeding competent Tau aggregates by endocytosis. a, b Effect of inhibition of lysosomal $\mathrm{H}^{+} \mathrm{ATPase}$ by Bafilomycin A1 (a) and permeabilization of acidic membrane compartments with LLOME (b) on seeding potency of TauRD aggregates formed with or without Clu. TauRD-YT cells were treated with increasing concentrations of the inhibitors in combination with 500 ng TauRD seeds or 200 ng TauRD/Clu seeds without a transfection reagent. FRET positive (pos.) cells were analyzed after $48 \mathrm{~h}$. Data represent the mean \pm SEM ( $n=5$ independent experiments). Significance is represented relative to control cells treated with the vehicle without Bafilomycin A1 or LLOME. ${ }^{\star \star} p<0.01,{ }^{\star \star \star} p<0.001$ by twoway ANOVA with Sidak post hoc test (TauRD vehicle vs. TauRD $10 \mathrm{nM}$ Bafilomycin A1 $p=2.9 \times 10^{-5}$; TauRD/Clu vehicle vs. TauRD/Clu $10 \mathrm{nM}$ Bafilomycin A1 $p=3.9 \times 10^{-4}$; TauRD vehicle vs. TauRD $500 \mu \mathrm{M}$ LLOME $p=4 \times 10^{-10}$; TauRD/Clu vehicle vs. TauRD/Clu $250 \mu M$ LLOME $p=0.002$; TauRD/Clu vehicle vs. TauRD/Clu $500 \mu \mathrm{M}$ LLOME $p<1 \times 10^{-15}$ ). c Heparan sulfate proteoglycan (HSPG) mediated internalization of TauRD and TauRD/ Clu aggregates. TauRD-YT cells were treated with increasing concentrations of heparin in combination with $1 \mu$ g TauRD seeds or TauRD/Clu seeds without a transfection reagent. FRET positive (pos.) cells were analyzed after $48 \mathrm{~h}$. Data represent the mean \pm SEM ( $n=4$ independent experiments). Significance is represented relative to control cells treated with the vehicle without heparin. ${ }^{\star \star} p<0.01,{ }^{\star \star \star} p<0.001$ by two-way ANOVA with Sidak post hoc test (TauRD vehicle vs. TauRD $2 \mu \mathrm{g} / \mathrm{ml}$ heparin $p=0.0017$; TauRD vehicle vs. TauRD $20 \mu \mathrm{g} / \mathrm{ml}$ heparin $p=3 \times 10^{-4}$; TauRD vehicle vs. TauRD $200 \mu \mathrm{g} / \mathrm{ml}$ heparin $p=2.5 \times 10^{-4}$; TauRD/Clu vehicle vs. TauRD/Clu $0.2 \mu \mathrm{g} / \mathrm{ml}$ heparin $p=4.4 \times 10^{-6}$; TauRD/Clu vehicle vs. TauRD/Clu $2 \mu \mathrm{g} / \mathrm{ml}$ heparin, TauRD/ Clu vehicle vs. TauRD/Clu $20 \mu \mathrm{g} / \mathrm{ml}$ heparin and TauRD/Clu vehicle vs. TauRD/Clu $200 \mu \mathrm{g} / \mathrm{ml}$ heparin $p<1 \times 10^{-15}$ ). d, e Colocalization of TauRD/Clu seeds (TauRD-A532 in green, Clu-A647 in red) (arrowheads) with the endocytosis marker CHMP2a (magenta) (d) and the marker of ruptured endomembranes, galectin-8 (GAL8; magenta) (e) in TauRD-T cells. A representative result of confocal imaging is shown. The cell outline is indicated by a white dashed line. Scale bar, $10 \mu \mathrm{m}$. ( $n=3$ independent experiments). f, $\mathbf{g}$ Colocalization of TauRD/Clu seeds with endogenous TauRD-mTurquoise2 (TauRD-mTurq) aggregates. $\mathbf{f}$ A representative slice from a confocal stack is shown (scale bar, $10 \mu \mathrm{m}$ ) where cells are outlined with a white dashed line. One aggregate region, marked with a square in the slice, is represented by volume rendering ( $1 \mu \mathrm{m}$ scale bars indicated by arrows). Channels are also displayed separately. TauRD-A532 seed in green, Clu-A647 in red, endogenous TauRD aggregate in turquoise. $(n=3$ independent experiments). g Quantification of relative fluorescence intensity in the aggregate shown in the inset. TauRD-mTurq (blue), TauRD-A532 (green) and Clu-A647 (red). The colocalization line profile on a mid focal plane (inset image) along the white arrow is shown. Scale bar, $1 \mu \mathrm{m}$.

undergoes prion-like aggregate propagation in Parkinson's disease $(\mathrm{PD})^{1}$. Clu also delayed the formation of ThT-positive aggregates of $\alpha$-Syn (with early-onset-PD mutation A53T), even at very low molar ratios to $a-S y n n^{14,64}$ (Fig. 5a). However, as in the case of Tau, $a$-Syn fibrils were nevertheless assembled (Supplementary Fig. 9a, b) and similar amounts of aggregates were generated in the absence and presence of Clu, as demonstrated by filtration assay (Supplementary Fig. 9b). Seeding competent $\alpha$-Syn species formed slightly before the accumulation of ThT-positive aggregates, as analyzed in HEK293T cells stably expressing GFPa-Syn(A53T) (Fig. 5a-c). Lipofectamine was used to render seed uptake independent of cellular machinery for internalization. In the absence of Clu, seeding with $\alpha$-Syn(A53T) resulted in $\sim 60 \%$ of cells with aggregates. Seeding was markedly suppressed by substoichiometric amounts of Clu at ratios of Clu: $\alpha-S y n$ of 1:1,000 to 1:100 (Fig. 5b, c). This effect was also confirmed with 
In vitro aggregation

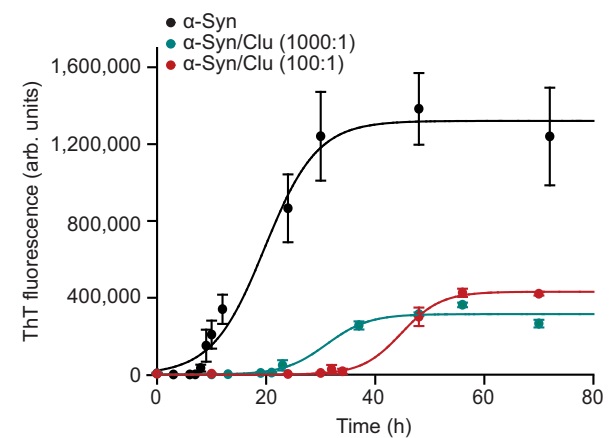

C

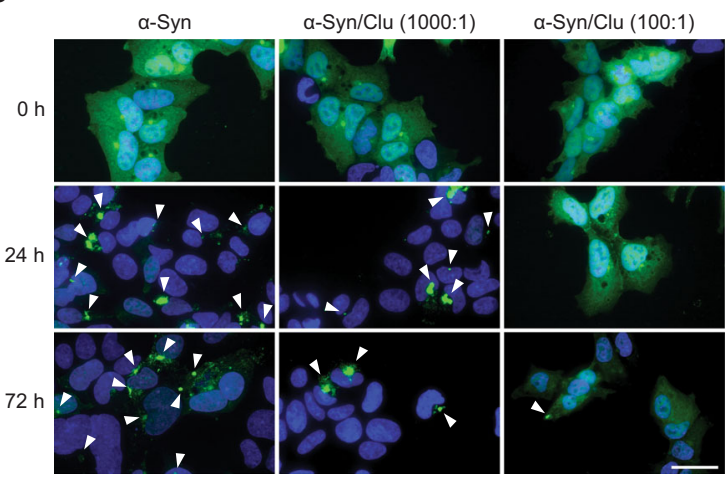

b

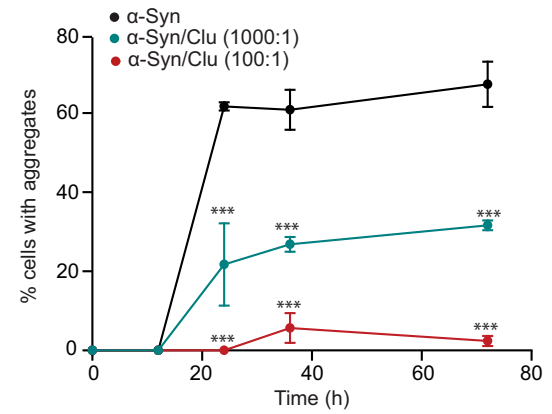

d Aggregate seeding in GFP-a-Syn(A53T) cells without transfection reagent

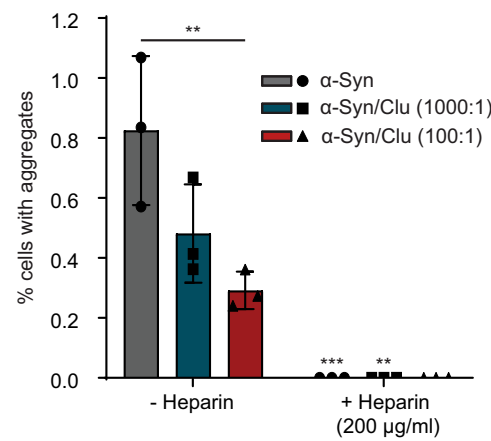

Fig. 5 Effect of Clusterin on $\boldsymbol{\alpha}$-Synuclein aggregation and seeding. a Aggregation of $\alpha$-Syn $(300 \mu \mathrm{M})$ without (black) or with Clu ( $0.3 \mu \mathrm{M}$ and $3 \mu \mathrm{M}$ in cyan and red, respectively) was monitored by ThT fluorescence. Molar ratios of $\alpha$-Syn:Clu are indicated. arb.units, arbitrary units. Averages \pm SEM ( $n=4$ independent experiments). $\mathbf{b}$ Effect of Clu on seeding potency of $\alpha$-Syn aggregation reactions as in (a). Seeding was measured by quantifying the fraction of HEK293T cells stably expressing GFP- $\alpha$-Syn(A53T) that contained aggregates after $24 \mathrm{~h}$ of seeding (200 ng $\alpha$-Syn) with lipofectamine. Molar ratios of $\alpha$ Syn:Clu are indicated. Significance is represented relative to $\alpha$-Syn alone at each time point. Averages \pm SEM ( $n=$ at least 100 cells examined over three independent experiments). ${ }^{\star \star \star} p<0.001$ by two-way ANOVA with Sidak post hoc test. ( $\alpha$-Syn $24 \mathrm{~h}$ vs. $\alpha$-Syn/Clu $1000: 124 \mathrm{~h} p=1.9 \times 10^{-8} ; \alpha$-Syn $24 \mathrm{~h}$ vs. $\alpha$-Syn/Clu 100:1 24 h $p=8.4 \times 10^{-13} ; \alpha$-Syn 36 h vs. $\alpha$-Syn/Clu 1000:1 36 h $p=4.5 \times 10^{-7} ; \alpha$-Syn 36 h vs. $\alpha$-Syn/Clu 100:1 36 h $p=1.3 \times 10^{-11} ; \alpha-S y n$ 72 h vs. $\alpha$-Syn/Clu 1000:1 $72 \mathrm{~h} p=1.8 \times 10^{-7} ; \alpha$-Syn $72 \mathrm{~h}$ vs. $\alpha$-Syn/Clu 100:1 $72 \mathrm{~h} p=2.3 \times 10^{-13}$ ). c Representative images of HEK 293 T GFP- $\alpha$-Syn(A53T) cells seeded with aggregation reactions (200 ng $\alpha$-Syn after $0 \mathrm{~h}, 24 \mathrm{~h}$ and $72 \mathrm{~h}$ aggregation (a)) with or without Clu. GFP- $\alpha$-Syn(A53T) and DAPI nuclear staining are shown in green and blue, respectively. Arrowheads indicate aggregates. Scale bar, $30 \mu \mathrm{m}$. d Heparan sulfate proteoglycan (HSPG) mediated internalization of $\alpha$-Syn and $\alpha$-Syn/Clu aggregates. Seeding was measured by quantifying the fraction of GFP- $\alpha$-Syn(A53T) cells that contained aggregates after $72 \mathrm{~h}$ of seeding ( $50 \mu \mathrm{g} \alpha$-Syn after $72 \mathrm{~h}$ aggregation (a)) without lipofectamine (-Heparin). GFP- $\alpha$-Syn(A53T) cells were also treated with $\alpha$-Syn and $\alpha$-Syn/Clu aggregates $(50 \mu \mathrm{g})$ in combination with heparin $(200 \mu \mathrm{g} / \mathrm{ml})$. Molar ratios of $\alpha$-Syn:Clu are indicated. Data represent the mean \pm SEM $(n=$ at least 1000 cells examined over three independent experiments). ${ }^{\star \star} p<0.01,{ }^{\star \star \star} p<0.001$ by two-way ANOVA with Sidak post hoc test ( - Heparin $\alpha$-Syn vs. - Heparin $\alpha$-Syn/Clu 100:1 $p=0.003 ;$ - Heparin $\alpha$-Syn vs. +Heparin $\alpha$-Syn $p=4.8 \times 10^{-5}$; - Heparin $\alpha$-Syn/Clu 1000:1 vs. +Heparin $\alpha$-Syn/Clu 1000:1 $p=0.007)$. The significance of + Heparin reactions is relative to the respective - Heparin control.

neuroblastoma SH-SY5Y cells stably expressing GFP- $\alpha$-Syn (A53T) (Supplementary Fig. 9c, d), however higher amounts of seeding material (10 $\mu$ g a-Syn for SH-SY5Y cells, $200 \mathrm{ng}$ a-Syn for HEK293T) were necessary to obtain comparable seeding efficiencies. The observed decrease in $\alpha$-Syn seeding in the presence of Clu does not appear to be due to reduced formation of insoluble fibrils (Supplementary Fig. 9b), but rather to changes in the effective concentration of other seeding competent species. Note that $\mathrm{Clu}$ increased the seeding potency of TauRD aggregates approximately twofold even when used at a low Clu:TauRD ratio of 1:100 (Supplementary Fig. 9e). Thus, while having similar effects on aggregation kinetics in vitro, Clu has opposite effects on the seeding activity of Tau and $\alpha$-Syn aggregates in the cellular assay, enhancing the former and suppressing the latter.

To test the effect of Clu on a-Syn seeding in cells with the unperturbed plasma membrane, we omitted the transfection reagent. Under these conditions, Clu still clearly suppressed $\alpha$-Syn seeding (Fig. 5d). Consistent with the notion that HSPGs are also involved in $\alpha$-Syn internalization ${ }^{57}$, aggregate seeding by $\alpha$-Syn and $a-S y n / C l u$ was completely suppressed in the presence of heparin $(200 \mu \mathrm{g} / \mathrm{ml}$, Fig. $5 \mathrm{~d})$. Thus, HSPGs participate in the internalization of both $\alpha$-Syn and TauRD seeds, independent of the presence of Clu.

Clusterin enhances Tau seeding and toxicity in neurons. To extend our findings to neuronal cells, we used primary mouse neurons transduced with TauRD (residues 244-372, P301L/ V337M) fused to YFP (TauRD-Y). We incubated neurons with either aggregates of TauRD, TauRD/Clu, or buffer control without transfection reagent and after 4 days monitored the formation of aggregates of endogenous TauRD-Y by fluorescence microscopy (Fig. 6a-c). Incubation with TauRD seeds alone induced the formation of TauRD-Y inclusions in $\sim 12 \%$ of neurons, while TauRD/Clu induced aggregation in $\sim 45 \%$ of neurons (Fig. $6 \mathrm{~b}, \mathrm{c}$ ). While there was no difference in neuronal viability 4 days after seeding, treatment with TauRD/Clu resulted in a $\sim 30 \%$ decrease in viability 7 days after seeding compared to cells seeded with 
a

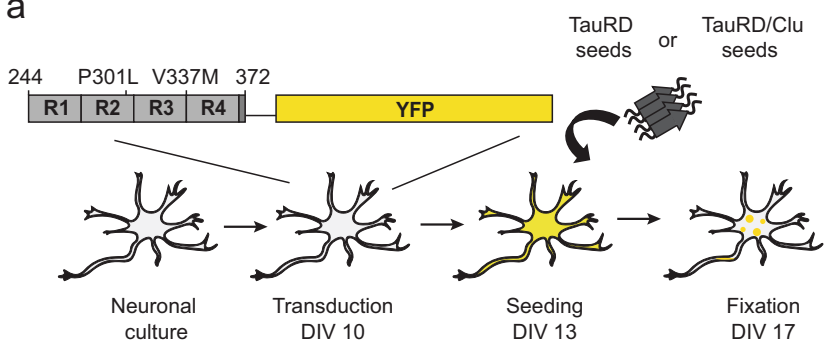

b

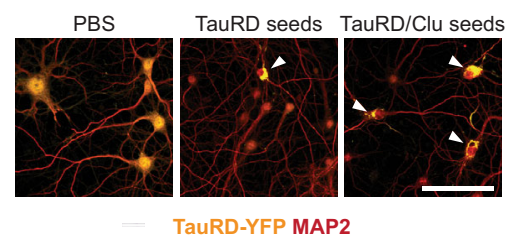

d

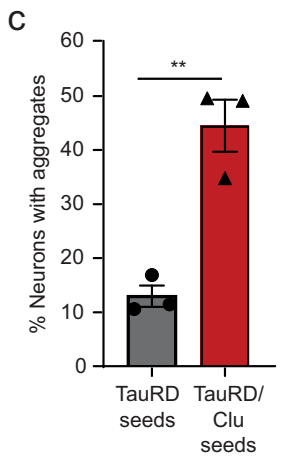

Fig. 6 Clusterin enhances Tau seeding and toxicity in neurons. a Workflow for TauRD aggregate seeding experiments with primary mouse neurons. DIV, days in vitro. b Representative fluorescence microscopy images of primary mouse neurons expressing TauRD-YFP (yellow) incubated with PBS, TauRD seeds or TauRD/Clu seeds (70 ng TauRD). Neurons were stained with an antibody against the neuronal marker MAP2 (red). Arrowheads indicate aggregates. Scale bar, $20 \mu \mathrm{m}$. c Comparison of seeding competence of TauRD (gray) and TauRD/Clu (red) seeds ( $70 \mathrm{ng}$ TauRD) in primary mouse neurons. The fraction of neurons containing YFPpositive aggregates by fluorescence microscopy imaging was quantified. Data represent the mean \pm SEM ( $n=$ at least 800 cells examined over 3 independent experiments). ${ }^{\star *} p<0.01(p=0.0038)$ by two-tailed Student's $t$-test. d Viability of neurons expressing TauRD-YFP at 4 and 7 days after incubation with TauRD (black) or TauRD/Clu (red) seeds (11 ng TauRD). Data from MTT assays are normalized to the control sample incubated with PBS (100\%) and represent the mean \pm SEM ( $n=5$ independent experiments). ${ }^{\star} p<0.05(p=0.034)$; n.s., not significant $(p=0.99)$ by twoway ANOVA with Sidak post hoc test. e Hypothetical model for the role of Clu (red) in amyloid seeding of Tau (brown) and $\alpha$-Synuclein ( $\alpha$-Syn) (blue). Tau and $\alpha$-Syn seeding competent species, partially produced by chaperone-mediated disaggregation, are released to the extracellular space from cells containing amyloid aggregates (gray). Clu (red) interacts with these species increasing seeding competence for Tau upon uptake by neighboring cells via the endosomal pathway. In contrast, Clu inhibits seeding for $\alpha$-Syn. Tau/Clu seeds efficiently template aggregation of endogenous Tau, resulting in cytotoxicity, while $\alpha$-Syn/Clu seeds are unable to template aggregation of endogenous $\alpha$-Syn.

intracellular chaperones such as the Hsp70 system ${ }^{65}$ may generate seeding competent Tau species, which could be a substrate for stabilization by Clu after release into the extracellular space (Fig. 6e). Although the exact mechanism by which Tau species are released from cells remains unclear ${ }^{7}$, seeding competent, highmolecular-weight Tau that could be acted on by Clu, has been detected in the cerebrospinal fluid of $\mathrm{AD}$ patients ${ }^{66}$. TauRD enters target cells in complex with Clu, apparently by endocytosis (Fig. 6e). However, TauRD/Clu seeds induce vesicle rupture and escape from endosomes to the cytosol, as described for Tau alone and other amyloidogenic proteins ${ }^{54,58}$ (Fig. 6e), enabling them to induce aggregation of endogenous Tau. Thus, Clu fails to mediate efficient lysosomal degradation of Tau, in contrast to other Clubound misfolded proteins ${ }^{31,32}$. Seed uptake and permeation of endolysosomal membranes may be facilitated by the relatively small size of the Tau species stabilized by Clu.

In contrast to a possible role of extracellular Clu in promoting Tau pathology, intracellular Clu, accumulating under stress conditions ${ }^{9}$, may be protective by interfering with de novo Tau aggregation. The latter activity would be consistent with a recent study reporting colocalization of Clu with intracellular Tau tangles in the brain of mice overexpressing human Tau (P301L) and exacerbated Tau pathology in CLU knock-out animals ${ }^{40}$. However, aggregate spreading was not explicitly assessed. It also remains to be investigated whether other apolipoproteins including ApoE, which also influences Tau pathology ${ }^{67-70}$, may compensate for the loss of extracellular $\mathrm{Clu}^{71-74}$. Whether Clu ultimately delays or promotes Tau pathology may depend on the stage of the disease and multiple other factors.

The seeding-enhancing effect of Clu appears to be Tau specific. In the case of $a$-Syn, another amyloidogenic protein that undergoes prion-like aggregate spreading ${ }^{1}$, Clu blocked both aggregation and seeding (Fig. 6e), consistent with recent observations $s^{64,75,76}$. Indeed, Clu is upregulated in PD and other synucleinopathies ${ }^{77}$. Interestingly, progressive $\mathrm{PD}$ in older patients is often associated with mixed brain pathologies, 
including Tau aggregation ${ }^{78,79}$, raising the possibility of a tug of war between protective functions of Clu and collateral damage incurred. Thus, the ability of Clu to modulate transcellular Tau aggregate seeding may be broadly relevant in understanding the progressive nature of neurodegenerative pathologies.

\section{Methods}

Plasmids. The N1-TauRD (P301L/V337M)-EYFP construct and the mTurquoise2N1 plasmid were gifts from Marc Diamond and Michael Davidson, respectively (Addgene $\# 54843^{80}$ ). The N1-TauRD (P301L/V337M)-mTurquoise2 plasmid for TauRD-mTurquoise2 expression in HEK293T cells was constructed by cloning the TauRD sequence (Tau amino acids 244-372, containing the diseaserelated mutations P301L and V337M) from N1-TauRD (P301L/V337M)-EYFP into the mTurquoise2N1 plasmid using the EcoRI and NheI restriction sites. The N1-FLTau (0N4R, P301L/V337M)-EYFP and N1-FLTau (0N4R, P301L/V337M)mTurquoise2 plasmids were constructed by substitution of the TauRD (P301L/ V337M) sequence from N1-TauRD (P301L/V337M)-EYFP and N1-TauRD (P301L/V337M)-mTurquoise2 plasmids by the FLTau (0N4R, P301L/V337M) sequence from the pRK5-EGFP-Tau P301L plasmid (a gift from Karen Ashe (Addgene \#46908 ${ }^{81}$ )) after introducing the mutation V337M, by Gibson assembly using the Gibson Assembly Master Mix (New England Biolabs). The linker between Tau and the fluorescent protein was composed of 21 amino acids in al cases (YPYGILQSTVPRARDPPVATA/M for YFP and mTurquoise2 plasmids, respectively) to avoid interference of fluorescent protein with fibril formation.

The pHUE-TauRD (P301L/V337M) plasmid was made by inserting the TauRD sequence (Tau amino acids 244-371) in pHUE ${ }^{82}$ by Gibson assembly using the Gibson Assembly Master Mix (New England Biolabs). Plasmid pHUE-TauRD (C291A/P301L/C322A/V337M) containing cysteine-free TauRD and pHUETauRD (I260C/C291A/P301L/C322A/V337M) containing TauRD-I260C for fluorescent labeling was constructed by mutagenesis of the pHUE-TauRD (P301L/ V337M) plasmid using the Q5 ${ }^{\oplus}$ Site-Directed Mutagenesis Kit (New England Biolabs).

The Tau/pET29b plasmid used for wild-type FLTau (2N4R) expression and purification was a gift from Peter Klein (Addgene plasmid \#16316 ${ }^{83}$ ).

$\mathrm{pFhSynW2}$ and the $\mathrm{pVsVg}$ packing plasmids used for lentivirus production were a gift from Dieter Edbauer. The psPAX2 packing plasmid, also used for lentivirus production, was a gift from Didier Trono (Addgene plasmid \#12260). pFhSynW2 TauRD (P301L/V337M)-EYFP used for TauRD-EYFP expression in mouse primary neurons was constructed by PCR amplification of the TauRD (P301L/V337M)-EYFP sequence from the N1-TauRD (P301L/V337M)-EYFP plasmid.

The $\mathrm{pB}-\mathrm{T}-\mathrm{PAF}$ vector was a gift from James Rini. The pB-T-PAF-CluStrep plasmid, which was used for Clusterin (Clu) (Clu followed by a strep tag, WSHPQFEK) expression and purification, was constructed by cloning the Clu cDNA sequence amplified from human embryonic kidney 293T (HEK293T) cells into the pB-T-PAF vector. RNA was extracted from the cell pellet using the RNeasy Mini kit (Qiagen). cDNA was then synthesized using the QuantiTect Reverse Transcription Kit (Qiagen) and the Clu cDNA amplified by PCR. The PCR product was then digested and subcloned into the $\mathrm{pB}-\mathrm{T}-\mathrm{PAF}$ vector.

Plasmid pT7-7 $\alpha$-Syn A53T for the expression and purification of recombinant $\alpha$-Syn was a gift from Hilal Lashuel (Addgene plasmid \#105727 ${ }^{84}$ ) and EGFP- $\alpha$ SynA53T plasmid for a-Syn expression in HEK293T and SH-SY5Y cells were a gift from David Rubinsztein (Addgene plasmid \#4082385).

Cell lines. A HEK293-EBNA suspension cell line $e^{86}$ stably expressing the recombinant protein Clusterin-Strep tag (HEK293E-CluStrep) was generated by using a piggyBac transposon-based expression system ${ }^{87}$ employing the $\mathrm{pB}-\mathrm{T}-\mathrm{PAF}-$ CluStrep plasmid.

The monoclonal HEK293T cell line stably expressing two different constructs, TauRD N-terminally fused to either EYFP or mTurquoise2 (TauRD-YT cell line) was generated by transfecting HEK293T cells with N1-TauRD (P301L/V337M)EYFP and N1-TauRD (P301L/V337M)-mTurquoise2 plasmids using lipofectamine 3000 (Thermo Fisher Scientific). Cells expressing the constructs were selected by $1 \mathrm{mg} \mathrm{ml}^{-1} \mathrm{G} 418$ antibiotic (Gibco) selection, and monoclonal cell lines were generated by isolating cells expressing both TauRD fusion proteins in a 96 wellplate by cell sorting with a BD FACS Aria III (BD Biosciences) following amplification. A monoclonal cell line allowing Tau aggregation to be monitored by flow cytometry (FRET signal detection) with high efficiency was selected. In a similar way, a monoclonal HEK293T cell line stably expressing FLTau (0N4R, P301L/V337M) fused to either EYFP or mTurquoise2 was generated (FLTau-YT cell line) using the N1-FLTau (0N4R, P301L/V337M)-EYFP and N1-FLTau (0N4R, $\mathrm{P} 301 \mathrm{~L} / \mathrm{V} 337 \mathrm{M}$ )-mTurquoise2 plasmids, as well as monoclonal cell lines expressing just one of the constructs, N1-TauRD (P301L/V337M)-EYFP construct (TauRD-Y cell line), N1-TauRD (P301L/V337M)-mTurquoise2 construct (TauRD-T cell line), N1-FLTau (0N4R, P301L/V337M)-EYFP construct (FLTau-Y cell line) and N1FLTau (0N4R, P301L/V337M)-mTurquoise2 construct (FLTau-T cell line).

The HEK293T and SH-SY5Y cell lines stably expressing EGFP- $\alpha-S y n(A 53 T)^{88}$ were generated by transfecting HEK293T and SH-SY5Y cells with the EGFP- $\alpha-$ SynA53T plasmid using lipofectamine (Thermo Fisher Scientific). Cells expressing the constructs were selected by 2000 and $1000 \mu \mathrm{g} \mathrm{ml}^{-1} \mathrm{G} 418$ antibiotic (Gibco) treatment, respectively. The SH-SY5Y EGFP- $\alpha$-Syn(A53T) cell line was enriched by selecting cells expressing EGFP- $\alpha$-SynA53T by cell sorting with a BD FACS Aria III (BD Biosciences) following amplification.

HEK293T and SH-SY5Y cell lines were maintained at $37^{\circ} \mathrm{C}$ and $5 \% \mathrm{CO}_{2}$ in Dulbecco's modified Eagle's medium (DMEM, Biochrom KG) supplemented with $10 \%$ fetal bovine serum (Gibco), $100 \mathrm{U} \mathrm{ml}^{-1}$ penicillin (Gibco), $100 \mu \mathrm{I} \mathrm{ml}{ }^{-1}$ streptomycin sulfate (Gibco) and $2 \mathrm{mM} \mathrm{L}$-glutamine (Gibco). Stable cell lines were maintained in the medium described above supplemented with G418 $\left(200 \mu \mathrm{g} \mathrm{ml}^{-1}\right)$

Primary neuronal cultures. Primary mouse neurons were prepared from $15.5 \mathrm{CD}$ 1 wild-type embryos. All experiments involving mice were performed in accordance with the relevant guidelines and regulations. Pregnant females were sacrificed by cervical dislocation, the uterus was removed from the abdominal cavity and placed into a $10 \mathrm{~cm}$ sterile Petri dish on ice containing dissection medium, consisting of Hanks' balanced salt solution (HBSS) supplemented with $0.01 \mathrm{M}$ HEPES, $0.01 \mathrm{M} \mathrm{MgSO}_{4}$, and 1\% Penicillin/Streptomycin. Each embryo was isolated, heads were quickly cut, and brains were removed from the skull and immersed in an ice-cold dissection medium. Cortical hemispheres were dissected and meninges were removed under a stereomicroscope. Cortical tissue from typically six to seven embryos was transferred to a $15 \mathrm{ml}$ sterile tube and digested with $0.25 \%$ trypsin containing $1 \mathrm{mM}$ ethylenediaminetetraacetic acid (EDTA) and $15 \mu \mathrm{l} 0.1 \%$ DNAse I for $20 \mathrm{~min}$ at $37^{\circ} \mathrm{C}$. The enzymatic digestion was stopped by removing the supernatant and washing the tissue twice with a Neurobasal medium (Invitrogen) containing 5\% FBS. The tissue was resuspended in a $2 \mathrm{ml}$ medium and triturated to achieve a single cell suspension. Cells were spun at $130 \times g$, the supernatant was removed, and the cell pellet was resuspended in a Neurobasal medium with 2\% B27 (Invitrogen), 1\% L-Glutamine (Invitrogen), and 1\% Penicillin/Streptomycin (Invitrogen). For immunofluorescence, neurons were cultured in 24-well plates on $13 \mathrm{~mm}$ coverslips coated with $1 \mathrm{mg} \mathrm{ml}^{-1}$ poly-D-Lysine (Sigma) and $1 \mu \mathrm{g} \mathrm{ml}^{-1}$ laminin (Thermo Fisher Scientific) $(100,000$ neurons per well). For viability measurements, neurons were cultured in 96 -well plates coated in the same way $(19,000$ neurons per well). Lentiviral transduction was performed at DIV 10. Viruses were thawed and immediately added to a freshly prepared neuronal culture medium. Neurons in 24-well plates received $1 \mu$ of virus per well. Neurons in 96-well plates received $0.15 \mu \mathrm{l}$ of virus per well. A fifth of the medium from cultured neurons was removed and the equivalent volume of virus-containing medium was added.

Lentivirus production. HEK293T cells for lentiviral packaging (Lenti-X 293T cell line, Takara) were expanded to $70-85 \%$ confluency in DMEM Glutamax $\left(+4.5 \mathrm{~g} \mathrm{l}^{-1} \mathrm{D}\right.$-Glucose, - Pyruvate) supplemented with 10\% FBS (Sigma), 1\% G418 (Gibco), 1\% NEAA (Thermo Fisher Scientific), and 1\% HEPES (Biomol). Only low passage cells were used. For lentiviral production, a three-layered T525 $\mathrm{cm}^{2}$ flask (Falcon) was seeded and cells were henceforth cultured in a medium without G418. On the following day, cells were transfected with the pFhSynW2 TauRD (P301L/ V337M)-EYFP expression plasmid and the packaging plasmids psPAX2 and pVsVg using TransIT-Lenti transfection reagent (Mirus). The transfection mix was incubated for $20 \mathrm{~min}$ at room temperature (RT) and in the meanwhile, cell medium was exchanged. In total, $10 \mathrm{ml}$ transfection mix was added to the flask and left overnight. The medium was exchanged on the next day. After $48-52 \mathrm{~h}$, the culture medium containing the viral particles was collected and centrifuged for $10 \mathrm{~min}$ at $1200 \times g$. The supernatant was filtered through $0.45 \mu \mathrm{m}$ pore size filters using $50 \mathrm{ml}$ syringes, and a Lenti-X concentrator was added (Takara). After overnight incubation at $4{ }^{\circ} \mathrm{C}$, samples were centrifuged at $1500 \times g$ for $45 \mathrm{~min}$ at $4{ }^{\circ} \mathrm{C}$, the supernatant was removed, and the virus pellet was resuspended in $600 \mu \mathrm{l}$ TBS-5 buffer $(50 \mathrm{mM}$ Tris- $\mathrm{HCl} \mathrm{pH} 7.8,130 \mathrm{mM} \mathrm{NaCl}, 10 \mathrm{mM} \mathrm{KCl}, 5 \mathrm{mM} \mathrm{MgCl}$ ). After aliquoting, the virus was stored at $-80^{\circ} \mathrm{C}$.

Chemicals and cell treatments. Bafilomycin A1 was purchased from InvivoGen. L-Leucyl-L-Leucine methyl ester (hydrochloride) (LLOME) was purchased from Cayman chemicals. Both compounds were dissolved in DMSO and small aliquots were stored at $-20^{\circ} \mathrm{C}$ until further use. For non-treated samples, DMSO alone was used as a control. Alexa Fluor 532 C5 maleimide and Alexa Fluor $647 \mathrm{~N}$ hydroxysuccinimide (NHS) ester were purchased from Thermo Fischer Scientific and freshly dissolved in DMSO before protein labeling. Heparin sodium salt from porcine intestinal mucosa was purchased from Merck (H3393).

\section{Sodium dodecyl sulfate-polyacrylamide gel electrophoresis (SDS-PAGE) and} immunoblotting. Protein samples were boiled in SDS-PAGE sample buffer for 5 min. Protein samples were separated by electrophoresis on NuPAGE $4-12 \%$ BisTris SDS gels (Thermo Fisher Scientific) using NuPAGE MES SDS running buffer (Thermo Fisher Scientific) at $180 \mathrm{~V}$. Coomassie blue staining was performed with InstantBlue (Merck). For immunoblotting, proteins were transferred at $70 \mathrm{~V}$ for $2 \mathrm{~h}$ onto a nitrocellulose membrane (GE Healthcare) using a wet electroblotting system (BIO-RAD). Membranes were blocked for at least $1 \mathrm{~h}$ with $0.05 \%$ TBS-Tween and 5\% low-fat milk. Immunodetection was performed using mouse monoclonal Tau/ Repeat Domain antibody (TECAN, 2B11), anti-Tau-1 antibody (non-phosphorylated Tau, clone PC1C6, Merck, MAB3420), Tau monoclonal antibody (TAU-5, 
Total Tau, Thermo Fisher Scientific, MA5-12808), phospho-Tau antibody (Ser202, Thr205, AT8, Thermo Fisher Scientific, MN1020), mouse monoclonal Clu- $\alpha$ antibody (Santa Cruz Biotechnology, sc-5289), recombinant anti-Clusterin antibody (EPR2911, abcam, ab92548) and anti-alpha-Synuclein antibody (LB509, abcam, ab27766). Conjugated goat-anti mouse immunoglobulin G (IgG)-horseradish peroxidase (HRP) (Merck, A4416) or anti-rabbit IgG (whole molecule)-peroxidase antibody produced in goat (Merck, A9169) were used as secondary antibodies. Immobilon Classico Western HRP substrate or Immobilon ECL Ultra Western HRP Substrate (Merck) were used for detection. Quantification by densitometry was performed with AIDA Image Analyzer v.4.27 (Elysia Raytest) or Image J (Rasband, W.S., National Institutes of Health, USA). Full scan blots are provided in the Source Data file.

Protein purification. Seed aggregates for addition to cells were generated with purified recombinant cysteine-free TauRD (Tau residues 244-371, C291A/P301L/ $\mathrm{C} 322 \mathrm{~A} / \mathrm{V} 337 \mathrm{M}$ ) to avoid the use of reducing agents that might interfere with Clu function (Supplementary Fig. 1a). Mutation of the two cysteines in TauRD avoids the formation of intramolecular disulfide bonds that slows fibril formation ${ }^{42}$. Cysteine-free TauRD and TauRD-I260C were expressed as N-terminal $\mathrm{His}_{6}{ }_{-}$-ubiquitin fusion proteins in Escherichia coli BL21(DE3) cells transformed with the respective pHUE-TauRD plasmids via IPTG induction. The cell pellet from a 21 culture was resuspended in $50 \mathrm{ml}$ lysis buffer ( $50 \mathrm{mM}$ PIPES-NaOH pH 6.5, $250 \mathrm{mM} \mathrm{NaCl}, 10 \mathrm{mM}$ imidazole, $2 \mathrm{mM} \beta$-mercaptoethanol ( $\beta \mathrm{ME})$ ) supplemented with $1 \mathrm{mg} \mathrm{ml}^{-1}$ lysozyme, Complete EDTA-free protease inhibitor cocktail (MERK) and benzonase, and incubated while gently shaking at $4{ }^{\circ} \mathrm{C}$ for $30 \mathrm{~min}$. Cells were lysed by ultrasonication, and the lysate was cleared by centrifugation $\left(1 \mathrm{~h}, 40,000 \times g\right.$ at $\left.4^{\circ} \mathrm{C}\right)$. The supernatant was loaded onto a Ni-NTA column equilibrated with lysis buffer. The column was washed with high salt buffer $(50 \mathrm{mM}$ PIPES- $\mathrm{NaOH}$ pH 6.5, $500 \mathrm{mM} \mathrm{NaCl}, 10 \mathrm{mM}$ imidazole, $2 \mathrm{mM} \beta \mathrm{ME}$ ) and wash buffer (50 mM PIPES-NaOH pH 6.5, $250 \mathrm{mM} \mathrm{NaCl}, 50 \mathrm{mM}$ imidazole, $2 \mathrm{mM}$ $\beta M E$ ), and $\mathrm{His}_{6}$-ubiquitin-TauRD was eluted with elution buffer (50 mM PIPES$\mathrm{NaOH}$ pH 6.5, $50 \mathrm{mM} \mathrm{NaCl}, 250 \mathrm{mM}$ imidazole, $2 \mathrm{mM} \beta$-ME). The eluted fractions were collected and the salt concentration was reduced by diluting the sample (1:5) with PIPES buffer (50 mM PIPES-NaOH pH 6.5, $2 \mathrm{mM} \beta \mathrm{ME}$ ), followed by incubation with Usp2 ubiquitin protease $(0.5 \mathrm{mg})$ overnight at $4{ }^{\circ} \mathrm{C}$ in order to cleave the $\mathrm{His}_{6}$-ubiquitin tag. The cleavage mixture was applied to a Source30S cation exchange column and the TauRD protein was eluted with a $0-500 \mathrm{mM} \mathrm{NaCl}$ gradient in PIPES buffer. The TauRD protein was further purified by size exclusion chromatography (SEC) on Superdex-75 in phosphate-buffered saline (PBS). For TauRD-I260C, the buffer used for SEC contained $1 \mathrm{mM}$ tris(2-carboxyethyl) phosphine (TCEP) in order to prevent the formation of disulfide bonds. Fractions containing pure protein were combined, aliquoted, and flash-frozen in liquid nitrogen for storage at $-80^{\circ} \mathrm{C}$. A more detailed protocol can be found here, https:// edmond.mpdl.mpg.de/imeji/collection/3psV1MQj0fmYu5KS.

Wild type FLTau (2N4R) was expressed in E. coli BL21(DE3) transformed with the Tau/pET29b plasmid via IPTG induction. The cell pellet from 61 of culture was resuspended in $180 \mathrm{ml}$ lysis2 buffer (50 mM MES- $\mathrm{NaOH}$ pH $6.8,20 \mathrm{mM} \mathrm{NaCl}$, $1 \mathrm{mM} \mathrm{MgCl} 2,5 \mathrm{mM} \mathrm{DTT}$ ), applied to a French press cell disruptor, and subsequently boiled for $20 \mathrm{~min}$. The lysate was cleared by centrifugation $(1 \mathrm{~h}$ $40,000 \times g$ at $4{ }^{\circ} \mathrm{C}$ ), and the supernatant was loaded onto a Source30S cation exchange column equilibrated with lysis 2 buffer. The protein was eluted with a $0-500 \mathrm{mM} \mathrm{NaCl}$ gradient and further purified by SEC on Sephacryl S-200 in $20 \mathrm{mM}$ MES-NaOH pH 6.8, $20 \mathrm{mM} \mathrm{NaCl}, 10 \%$ glycerol. Fractions containing pure protein were combined, aliquoted, and flash-frozen in liquid nitrogen for storage at $-80^{\circ} \mathrm{C}$.

Recombinant Clu (CluStrep) was purified as described (https://doi.org/10.175 04/protocols.io.bvvkn64w). Strep-tagged Clu was expressed and secreted by HEK293E-CluStrep cells cultured in FreeStyle 293 Expression Medium (Thermo Fisher Scientific) for 4 days. The conditioned medium was then separated from the cells by centrifugation. For chromatographic purification, the medium was first dialyzed against wash buffer $(20 \mathrm{mM} \mathrm{Na}$ acetate $\mathrm{pH}$ 5.0). After removal of the precipitate by centrifugation, the supernatant was passed over a HiTrap SP XL cation exchange column. The column was washed with 10 column volumes (CV) denaturing buffer ( $20 \mathrm{mM} \mathrm{Na}$ acetate $\mathrm{pH} 5.0,6 \mathrm{M}$ urea), followed by $5 \mathrm{CVs}$ wash buffer. For protein elution, a $0-500 \mathrm{mM} \mathrm{NaCl}$ gradient in wash buffer was applied. Clu-containing fractions were further purified by SEC on Superdex-200 in $20 \mathrm{mM}$ $\mathrm{Na}$ acetate $\mathrm{pH} 5.0,100 \mathrm{mM} \mathrm{NaCl}, 1 \mathrm{mM}$ EDTA. Fractions containing pure $\mathrm{Clu}$ were concentrated, and the buffer was exchanged to PBS using a Nap5 (GE Healthcare) column. Aliquots were flash-frozen in liquid nitrogen for storage at $-80^{\circ} \mathrm{C}$

Recombinant human $\alpha$-Synuclein ( $\alpha$-Syn, A53T) was purified essentially as described ${ }^{89}$ (https://doi.org/10.17504/protocols.io.btynnpve). In brief, E. coli BL21 (DE3) cells were transformed with the pT7-7 $\alpha$-Syn A53T plasmid. Protein expression was induced with $1 \mathrm{mM}$ IPTG for $4 \mathrm{~h}$ at $37^{\circ} \mathrm{C}$. Bacteria were harvested and the pellet was lysed in high salt buffer $(750 \mathrm{mM} \mathrm{NaCl}, 50 \mathrm{mM}$ Tris- $\mathrm{HCl} \mathrm{pH} \mathrm{7.6,}$ $1 \mathrm{mM}$ EDTA). The lysate was sonicated for $5 \mathrm{~min}$ and boiled subsequently. The boiled suspension was centrifuged, the supernatant dialyzed against $50 \mathrm{mM} \mathrm{NaCl}$, $10 \mathrm{mM}$ Tris-HCl pH 7.6, and $1 \mathrm{mM}$ EDTA and purified by SEC on Superdex 200 in the same buffer. Fractions were collected and those containing $\alpha$-Syn were combined. The combined fractions were applied onto an anion exchange column (MonoQ). $\alpha$-Syn was purified by a gradient ranging from $50 \mathrm{mM}$ to $1 \mathrm{M} \mathrm{NaCl}$.
Fractions containing $\alpha$-Syn were combined and dialyzed in $150 \mathrm{mM} \mathrm{KCl}, 50 \mathrm{mM}$ Tris- $\mathrm{HCl} \mathrm{pH} \mathrm{7.6.} \mathrm{Aliquots} \mathrm{were} \mathrm{stored} \mathrm{at}-80^{\circ} \mathrm{C}$.

Deglycosylation. Purified Clu was deglycosylated with PNGase F (glycerol free, $\mathrm{NEB}$ ), following the manufacturer's instructions.

Rhodanese aggregation assay. Rhodanese $(100 \mu \mathrm{M})$ was denatured in $6 \mathrm{M}$ guanidinium- $\mathrm{HCl}, 5 \mathrm{mM}$ DTT for $1 \mathrm{~h}$ at $25^{\circ} \mathrm{C}$, and diluted 200-fold into PBS in the absence or presence of Clu $(0.5 \mu \mathrm{M})$. Bovine serum albumin (BSA) (Thermo Fisher Scientific) $(0.5 \mu \mathrm{M})$ was used as control. Aggregation was monitored immediately after dilution by measuring turbidity at $320 \mathrm{~nm}$ wavelength.

Filter retardation assay. Different amounts of $\alpha$-Syn aggregation reaction were diluted in PBS and subsequently applied onto a pre-wetted $0.2 \mu \mathrm{m}$ pore size cellulose acetate membrane in a Hoefer slot-blot apparatus. The membrane was subsequently washed twice with $0.1 \%$ Triton-X 100 . Immunodetection was performed as described above (Sodium dodecyl sulfate-polyacrylamide gel electrophoresis (SDS-PAGE) and immunoblotting).

Protein labeling. TauRD-I260C and Clu were labeled with Alexa532 C5 maleimide and Alexa647 NHS ester (Thermo Fisher Scientific), respectively. Before the labeling reaction, TauRD-I260C was incubated on ice in the purification buffer containing $2 \mathrm{mM}$ TCEP to reduce the cysteine residue, followed by removal of TCEP by SEC using a Nap5 (GE Healthcare) column pre-equilibrated with PBS buffer. Labeling of TauRD-I260C at an equimolar ratio of the fluorophore was performed in the PBS buffer for $1 \mathrm{~h}$ at RT. For the labeling reaction of Clu, PBS buffer was exchanged with $0.1 \mathrm{M}$ sodium bicarbonate buffer $(\mathrm{pH}$ 8.3) (N-terminal labeling buffer) using a Nap5 column, and labeling was subsequently performed at a twofold molar excess of fluorophore for $1 \mathrm{~h}$ at RT. Free dyes were removed using a Nap5 column, pre-equilibrated with PBS buffer. The labeling efficiency was measured by nanodrop and was typically about $65-70 \%$.

Fluorescence cross-correlation spectroscopy (dcFCCS). dcFCCS measurements were performed on a Micro Time 200 inverse time-resolved fluorescence microscope (PicoQuant) as described previously ${ }^{90,91}$. Samples were diluted 100 -fold in PBS (from $1 \mu \mathrm{M}$ to $10 \mathrm{nM}$ each labeled protein) immediately before each measurement. Autocorrelation was recorded for $30 \mathrm{~min}$. The diffusion time was obtained by fitting the curves with the triplet diffusion equation using Symphotime 64 (PicoQuant). The confocal volume $\left(V_{e f f}\right)$ was calibrated daily using Atto655 maleimide dye.

Estimation of the average molecular weight of Tau/Clu complexes. To estimate the average size of Tau/Clu complexes, we performed FCS measurements to determine the diffusion coefficient $(D)$. Diffusion coefficients $(D)$ were converted into hydrodynamic radii $\left(R_{H}\right)$ via the Stokes-Einstein equation (Eq. (1)), where $k_{\mathrm{B}}$ is the Boltzmann constant, $T$ is the temperature (in K) and $\eta$ is the solvent viscosity. Second, the correlation between $R_{\mathrm{H}}$ and the chain length (in amino acid residues, N) (Eq. (2)) for elongated proteins was applied ${ }^{92}$, and this chain length was converted to molecular weight by assuming an average molar mass for amino acids of $m_{\mathrm{aa}}=113 \mathrm{~g} / \mathrm{mol}$ (relative to amino acid abundance in eukaryotic proteins).

$$
R_{\mathrm{H}}=\left(\frac{k_{\mathrm{B}} T}{6 \pi \eta D}\right)
$$

$$
R_{\mathrm{H}}=(2.21 \pm 1.07) N^{0.57 \pm 0.02}
$$

Protein aggregation reactions and thioflavin-T (ThT) fluorescence measurements. Tau aggregation: $100 \mu \mathrm{l}$ of $10 \mu \mathrm{M}$ Tau (TauRD or FLTau), $2.5 \mu \mathrm{M}$ heparin, $2 \mathrm{mM} \mathrm{MgCl}_{2}$ were incubated in the presence or absence of Clu at $37^{\circ} \mathrm{C}$ with constant agitation $(850 \mathrm{rpm})$ in a thermomixer (Eppendorf). Aliquots were removed at the indicated time points, flash-frozen in liquid nitrogen, and stored at $-80^{\circ} \mathrm{C}$ until measurement of ThT fluorescence and seeding activity.

$\alpha$-Syn aggregation: purified $\alpha$-Syn $\left(5 \mathrm{mg} \mathrm{ml}^{-1}, 330 \mu \mathrm{M}\right)$ was centrifuged at $100,000 \times g$ for $1 \mathrm{~h}$. The supernatant was transferred into a new reaction tube and incubated in the presence or absence of Clu with constant agitation $(1000 \mathrm{rpm})$ at $37^{\circ} \mathrm{C}$ in a thermomixer. Aliquots were removed at the indicated time points and stored at $-80^{\circ} \mathrm{C}$ until measurement of ThT fluorescence and seeding activity.

For monitoring amyloid formation by ThT fluorescence, aliquots from aggregation reactions of Tau or $a$-Syn were diluted 50 or 100 -fold, respectively, into $20 \mu \mathrm{M}$ ThT in PBS. Excitation and emission wavelengths were $440 \mathrm{~nm}$ and $480 \mathrm{~nm}$, respectively. Measurements were performed with a FluoroMax-4 Spectrophotometer (HORIBA) using FluorEssence V3.9 (HORIBA). The emission signal was corrected with the reference signal of the lamp (S1/R1 wavelength, S: emission signal, R: reference signal) and by subtraction of the minimum data point ThT kinetics were fitted using Sigma plot 14.0 (Sigmoidal dynamic fitting, sigmoid 3 parameter equation). A more detailed protocol can be found here https:// edmond.mpdl.mpg.de/imeji/collection/3psV1MQj0fmYu5KS. 
Fractionation of in vitro aggregation reactions. TauRD and TauRD/Clu aggregation reactions were centrifuged at $16,100 \times g$ and $4{ }^{\circ} \mathrm{C}$ for $1 \mathrm{~h}$. The supernatant was collected. The pellet was washed with PBS, centrifuged at $16,100 \times g$ and $4{ }^{\circ} \mathrm{C}$ for $30 \mathrm{~min}$, and the resulting pellet was resuspended in the initial volume of PBS. The Tau and Clu content was quantified by immunoblotting.

Cell-based seeding assays. Seeding of HEK293T cells: 100,000 cells per well of the HEK293T reporter cell line (TauRD-YT or FLTau-YT) were dispensed into a 12 -well plate. For subsequent fluorescence microscopy imaging, a coverslip was placed on the well. In total, 16-24 h later, Tau aggregates were transfected with lipofectamine 3000 (Thermo Fisher Scientific). Specifically, aggregate samples were mixed with a mixture of $50 \mu \mathrm{l}$ Opti-MEM (Gibco) and $1.6 \mu \mathrm{l}$ lipofectamine 3000 reagents (Thermo Fisher Scientific) and incubated for $20 \mathrm{~min}$ at RT. The mixtures were added to the cells with $0.5 \mathrm{ml}$ of fresh medium. When lipofectamine was not used, the aggregates were mixed with $0.5 \mathrm{ml}$ of fresh medium and added directly to the cells replacing the medium. After 16-20 h (when using lipofectamine) or $48 \mathrm{~h}$ (without lipofectamine or when using the FLTau-YT reporter cells), cells were processed for FRET signal analysis by flow cytometry or fluorescence microscopy imaging. When heparin was used to block HSPGs receptors, $1 \mu \mathrm{g}$ of TauRD or TauRD/Clu aggregates were pre-incubated with different concentrations of heparin in $0.5 \mathrm{ml}$ medium for $16 \mathrm{~h}$ at $4{ }^{\circ} \mathrm{C}^{57}$. After incubation, mixtures were added to the cells by exchanging the medium and $48 \mathrm{~h}$ later the FRET signal of seeded aggregates was analyzed by flow cytometry.

Quantification of FRET positive cells by flow cytometry: cells were harvested with TrypL Express Enzyme (Gibco), washed with PBS once, and resuspended in PBS for analysis with an Attune NxT flow cytometer (Thermo Fisher Scientific). To measure the mTurquoise2 and FRET fluorescence signals, cells were excited with $405 \mathrm{~nm}$ laser light and fluorescence was determined using 440/50 and 530/30 filters, respectively. To measure the YFP fluorescence signal, cells were excited at $488 \mathrm{~nm}$ and emission was recorded using a 530/30 filter. For each sample, 50,000 single cells were analyzed. Data processing was performed using FlowJo V9 and V10.7.1 software (FlowJo LLC). After gating single cells, an additional gate was introduced to exclude YFP-only cells that show a false-positive signal in the FRET channel due to excitation at $405 \mathrm{~nm}^{93}$. The FRET positive gate was set by plotting the FRET fluorescence signal versus the mTurquoise2 fluorescence signal using as reference non-seeded cells (Supplementary Fig. 10).A more detailed protocol and the original .fcs files can be found here, https://edmond.mpdl.mpg.de/imeji/ collection/3psV1MQj0fmYu5KS0

Fluorescence microscopy imaging: cells were fixed with $4 \%$ paraformaldehyde (PFA) in PBS for 10 min, washed with PBS, and permeabilized with $0.1 \%$ TritonX100/PBS for 5 min. Nuclei were stained with NucBlue Fixed Cell ReadyProbes Reagent (Thermo Fisher Scientific) following the manufacturer's instructions, and the coverslips were mounted with Dako fluorescence mounting medium (Agilent). Confocal imaging was performed as described below (immunofluorescence microscopy).

When lysates from cells containing aggregates were used as seeding material, cell pellets were lysed with $0.05 \%$ Triton X-100/PBS, Complete EDTA-free protease inhibitor cocktail (MERK) and benzonase for $20 \mathrm{~min}$ on ice, total protein was quantified by Bio-Rad Protein Assay (Bio-Rad) and the amount of Tau protein was quantified by SDS-PAGE and immunoblotting using purified Tau as standard.

Seeding of primary neurons: $70 \mathrm{ng}$ of TauRD aggregates mixed with fresh medium (one-tenth of the medium in the well) were directly added to the neuronal cultures in 24-well plates at DIV 13. After 4 days of incubation (DIV 17), coverslips were processed as described below (immunofluorescence microscopy).

Seeding of $a$-Syn aggregation: HEK293T and SH-SY5Y cells expressing EGFP$\alpha$-Syn(A53T) were seeded in a 24-well plate containing a coverslip and $\alpha$-Syn aggregates were transfected after $24 \mathrm{~h}$ using lipofectamine 2000 (Thermo Fisher Scientific). For HEK293T cells, seed material containing $200 \mathrm{ng}$ of $\alpha$-Syn was diluted into a mixture of $25 \mu \mathrm{l}$ Opti-MEM (Gibco) and $1.5 \mu \mathrm{l}$ lipofectamine. Subsequently, the suspension was added to $0.5 \mathrm{ml}$ of cell culture. For SH-SY5Y cells, seed material containing $10 \mu \mathrm{g}$ of $\alpha$-Syn was diluted into a mixture of $25 \mu \mathrm{l}$ of Opti-MEM (Gibco) and $1.5 \mu \mathrm{l}$ of lipofectamine. Subsequently, the suspension was added to $0.5 \mathrm{ml}$ of cell culture. After $24 \mathrm{~h}$ cells were processed for fluorescence microscopy imaging as described below and confocal imaging was performed (immunofluorescence microscopy). When heparin was used to block HSPGs receptors in seeding experiments without lipofectamine, $50 \mu \mathrm{g}$ of $\alpha$-Syn or $\alpha$-Syn/ Clu aggregates were pre-incubated with or without heparin $(200 \mu \mathrm{g} / \mathrm{ml})$ in $0.5 \mathrm{ml}$ medium for $2 \mathrm{~h}$ at room temperature, followed by addition to the cells by the medium exchange. The medium was replaced after $24 \mathrm{~h}$ to limit the toxicity otherwise observed with $50 \mu \mathrm{g}$ of $\alpha$-Syn. After further $48 \mathrm{~h}$ cells were processed for fluorescence microscopy as described below and confocal imaging was performed (immunofluorescence microscopy).

Amyloid staining. Cells were fixed with $4 \% \mathrm{PFA} / \mathrm{PBS}$ for $10 \mathrm{~min}$, washed with PBS, and permeabilized with $0.25 \%$ Triton-X100/PBS for $30 \mathrm{~min}$. Coverslips were incubated with $1 \mu \mathrm{M} \mathrm{X}-34$ (Sigma) in 60\% PBS, 39\% ethanol, $0.02 \mathrm{M} \mathrm{NaOH}$ for 15 min, washed three times with $60 \%$ PBS, $39 \%$ ethanol, $0.02 \mathrm{M} \mathrm{NaOH}$ followed by two washes with PBS, and mounted with Dako fluorescence mounting medium (Agilent).
Immunofluorescence microscopy. Cells were fixed with 4\% PFA/PBS for 10 min, washed with PBS, and permeabilized with $0.1 \%$ Triton-X100/PBS for $5 \mathrm{~min}$. Blocking solution (8\% BSA/PBS) was added for $30 \mathrm{~min}$. Coverslips were transferred to a humid chamber and incubated overnight with the primary antibody diluted in 1\% BSA/PBS (anti-phospho-Tau AT8, MN1020, Thermo Fisher Scientific, 1:100 dilution; anti-CHMP2A, 10477-1-AP, Proteintech, 1:50 dilution; anti-Galectin 8, ab109519, Abcam, 1:50 dilution). Cells were then washed with 0.1\% Tween-20/ PBS, incubated with the corresponding secondary antibody ( $\mathrm{F}(\mathrm{ab}$ ')2-Goat antiMouse IgG Alexa Fluor 633 (A-21053); goat-anti Rabbit IgG Alexa Fluor 405 (A31556), Thermo Fisher Scientific) diluted in 1\% BSA/PBS (1:500) for $1 \mathrm{~h}$, washed with $0.1 \%$ Tween-20/PBS and stained with NucBlue Fixed Cell ReadyProbes Reagent (Thermo Fisher Scientific) in the case of using anti-phospho-Tau AT8 antibody. Coverslips were mounted with Dako fluorescence mounting medium (Agilent). The confocal imaging was performed at the Imaging Facility of Max Planck Institute of Biochemistry, Martinsried, on a LEICA TCS SP8 AOBS confocal laser scanning microscope (Wetzlar, Germany) equipped with a LEICA HCX PL APO 63×/NA1.4 oil immersion objective. Images were analyzed with Image J (Rasband, W.S., National Institutes of Health, USA).

To detect colocalization of TauRD-A532/Clu-A647 with endogenous TauRDmTurq aggregates, a series of $z$-stack images were acquired and then deconvolved using Huygens Essentials 19.10 (Scientific Volume Imaging). Three-dimensional volume renderings were generated using Volocity V6.3 (Quorum Technologies).

Aggregate formation in primary neurons: neurons were fixed at DIV 17 with $4 \%$ PFA/PBS for $20 \mathrm{~min}$; remaining free groups of PFA were blocked with $50 \mathrm{mM}$ ammonium chloride in PBS for 10 min at RT. Cells were rinsed once with PBS and permeabilized with $0.25 \%$ Triton X-100/PBS for $5 \mathrm{~min}$. After washing with PBS, a blocking solution consisting of $2 \%$ BSA (w/v) (Roth) and $4 \%$ donkey serum (v/v) (Jackson Immunoresearch Laboratories) in PBS was added for $30 \mathrm{~min}$ at RT. Coverslips were transferred to a light-protected humid chamber and incubated in anti-MAP2 (NB300-213, Novus Biologicals) primary antibody diluted in blocking solution (1:500) for $1 \mathrm{~h}$. Cells were washed with PBS and incubated with the secondary antibody Alexa Fluor 647 AffiniPure Donkey Anti-Chicken IgY (IgG) (703-605-155, Jackson Immunoresearch Laboratories) diluted 1:250 in blocking solution, with $0.5 \mu \mathrm{g} \mathrm{ml}^{-1}$ DAPI added to stain the nuclei. Coverslips were mounted on Menzer glass slides using Prolong Glass fluorescence mounting medium. Confocal images were obtained at an SP8 confocal microscope (Leica).

In the case of $\alpha$-Syn seeding experiments, HEK293T and SH-SY5Y cells were imaged at a CorrSight microscope (Thermo Fisher) in spinning disc mode with a $63 \mathrm{x}$ oil immersion objective. Images were acquired with MAPS software (Thermo Fisher) and afterward analyzed by Image J. Cells were counted manually and $\alpha$-Syn accumulations with high fluorescence intensity and a diameter larger than $500 \mathrm{~nm}$ were considered aggregates. Fractions containing aggregates were calculated by using Origin Pro 2019b.

Immunoprecipitation. FLTau-YT cells were lysed with $0.05 \%$ Triton X-100/PBS with Complete, Mini, EDTA-free Protease Inhibitor Cocktail (Merck), PhosSTOP (Merck), and benzonase for $20 \mathrm{~min}$ on ice. Total protein was quantified (Bio-Rad protein assay) and the amount of FLTau protein was quantified by SDS-PAGE and quantitative immunoblotting with purified FLTau as standard. Lysates were diluted in PBS ( $400 \mu \mathrm{l}$ at $3 \mathrm{mg} / \mathrm{ml}$ total protein) and incubated with or without Clu (molar ratio FLTauYT:Clu 1:1) for $16 \mathrm{~h}$ at $37^{\circ} \mathrm{C}$. Immunoprecipitation was then performed with Dynabeads Protein G Immunoprecipitation Kit (Sigma-Aldrich) following the manufacturer's instructions. Briefly, $25 \mu \mathrm{l}$ of magnetic beads were incubated with $5 \mu \mathrm{g}$ of phospho-Tau antibody (AT8 antibody, MN1020, Thermo Fisher Scientific) in antibody binding and washing buffer with rotation for $30 \mathrm{~min}$ at room temperature. The beads were then washed and incubated with cell lysate with rotation for $1.5 \mathrm{~h}$ at room temperature. After incubation, the beads were washed 3 times with washing buffer, transferred to a clean tube, and elution was performed by addition of $40 \mu \mathrm{l}$ sample buffer $/ 100 \mathrm{mM}$ DTT to the beads and incubation at $70^{\circ} \mathrm{C}$ for $10 \mathrm{~min} .5 \mu \mathrm{l}$ of $1.5 \mathrm{M}$ Tris $\mathrm{pH} 8.8$ was added to the elution after removal of the magnetic beads. Samples $(13 \mu$ l of eluate and $2 \mu$ lof lysate as input) were subsequently analyzed by SDS-PAGE and immunoblotting as described above (Sodium dodecyl sulfate-polyacrylamide gel electrophoresis (SDSPAGE) and immunoblotting).

Neuronal viability measurements. At DIV 13, $11 \mathrm{ng}$ of TauRD aggregates mixed with fresh medium (one-tenth of the medium in the well) were directly added to the neuronal cultures in 96-well plates. Viability was determined with the MTT assay using thiazolyl blue tetrazolium bromide (MTT) reagent (Sigma-Aldrich). The cell medium was exchanged for $100 \mu \mathrm{l}$ of fresh medium. Subsequently, $20 \mu \mathrm{l}$ of $5 \mathrm{mg} \mathrm{ml}^{-1}$ MTT in PBS was added and incubated for $2-4 \mathrm{~h}$ at $37^{\circ} \mathrm{C}, 5 \% \mathrm{CO}_{2}$. Subsequently, $100 \mu \mathrm{l}$ solubilizer solution (10\% SDS, $45 \%$ dimethylformamide in water, $\mathrm{pH}$ 4.5) was added, and on the following day, absorbance was measured at $570 \mathrm{~nm}$. Each condition was measured in six replicates per experiment and absorbance values were averaged for each experiment.

Electron microscopy. For negative stain analysis, continuous carbon grids (Quantfoil) were glow discharged using a plasma cleaner (PDC-3XG, Harrick) for 30 s. Grids were incubated for 5 min with Tau samples, blotted and stained with 
$0.5 \%$ uranyl acetate solution, dried, and imaged in a Titan Halo (FEI) transmission electron microscope using SerialEM.

In the case of $\alpha$-Syn, grids were incubated for 1 min with the samples, blotted, and subsequently washed two times with water for $10 \mathrm{~s}$. The blotted grids were stained with $0.5 \%$ uranyl acetate solution, dried, and imaged in a Polara cryoelectron microscope (FEI) at $300 \mathrm{kV}$ using SerialEM.

Statistical analysis. Statistical analysis was performed with Sigma Plot 14.0 or GraphPrism6. Sample size ( $n$ ) given in figure legends describe measurements taken from distinct, independent samples. Normality was assessed in all cases. Logtransformation was applied on Bafilomycin treatment data to conform to normal distribution prior to statistical analysis. A two-tailed Student's $t$-test was used for simple comparisons. One-way ANOVA with Bonferroni post hoc test or Two-way ANOVA with Sidak post hoc test was used for multiple comparisons.

Reporting summary. Further information on research design is available in the Nature Research Reporting Summary linked to this article.

\section{Data availability}

All data supporting the findings of this study are available within the manuscript. Other data are available from the corresponding author upon reasonable request. Source data are provided with this paper.

Received: 26 January 2021; Accepted: 16 July 2021;

Published online: 11 August 2021

\section{References}

1. Peng, C., Trojanowski, J. Q. \& Lee, V. M. Protein transmission in neurodegenerative disease. Nat. Rev. Neurol. 16, 199-212 (2020).

2. Jucker, M. \& Walker, L. C. Propagation and spread of pathogenic protein assemblies in neurodegenerative diseases. Nat. Neurosci. 21, 1341-1349 (2018).

3. Vaquer-Alicea, J. \& Diamond, M. I. Propagation of protein aggregation in neurodegenerative diseases. Annu. Rev. Biochem. 88, 785-810 (2019).

4. Iadanza, M. G., Jackson, M. P., Hewitt, E. W., Ranson, N. A. \& Radford, S. E. A new era for understanding amyloid structures and disease. Nat. Rev. Mol. Cell Biol. 19, 755-773 (2018).

5. Sibilla, C. \& Bertolotti, A. Prion properties of SOD1 in amyotrophic lateral sclerosis and potential therapy. Cold Spring Harb Perspect Biol 9, https://doi. org/10.1101/cshperspect.a024141 (2017).

6. Wang, Y. et al. The release and trans-synaptic transmission of Tau via exosomes. Mol. Neurodegener. 12, 5 (2017).

7. Brunello, C. A., Merezhko, M., Uronen, R. L. \& Huttunen, H. J. Mechanisms of secretion and spreading of pathological tau protein. Cell Mol. Life Sci. 77, 1721-1744 (2020).

8. Chaplot, K., Jarvela, T. S. \& Lindberg, I. Secreted chaperones in neurodegeneration. Front Aging Neurosci. 12, 268 (2020).

9. Foster, E. M., Dangla-Valls, A., Lovestone, S., Ribe, E. M. \& Buckley, N. J. Clusterin in Alzheimer's disease: mechanisms, genetics, and lessons from other pathologies. Front. Neurosci. 13, 164 (2019).

10. Wyatt, A. R., Yerbury, J. J., Ecroyd, H. \& Wilson, M. R. Extracellular chaperones and proteostasis. Annu Rev. Biochem 82, 295-322 (2013).

11. Lee, K. W. et al. Clusterin regulates transthyretin amyloidosis. Biochem. Biophys. Res. Commun. 388, 256-260 (2009).

12. Narayan, P. et al. The extracellular chaperone clusterin sequesters oligomeric forms of the amyloid-beta(1-40) peptide. Nat. Struct. Mol. Biol. 19, 79-83 (2011).

13. Gregory, J. M. et al. Clusterin protects neurons against intracellular proteotoxicity. Acta Neuropathol. Commun. 5, 81 (2017).

14. Yerbury, J. J. et al. The extracellular chaperone clusterin influences amyloid formation and toxicity by interacting with prefibrillar structures. FASEB J. 21, 2312-2322 (2007).

15. Yu, J. T. \& Tan, L. The role of clusterin in Alzheimer's disease: pathways, pathogenesis, and therapy. Mol. Neurobiol. 45, 314-326 (2012).

16. Lambert, J. C. et al. Genome-wide association study identifies variants at CLU and CR1 associated with Alzheimer's disease. Nat. Genet. 41, 1094-1099 (2009).

17. Harold, D. et al. Genome-wide association study identifies variants at CLU and PICALM associated with Alzheimer's disease. Nat. Genet. 41, 1088-1093 (2009).

18. DeMattos, R. B. et al. Clusterin promotes amyloid plaque formation and is critical for neuritic toxicity in a mouse model of Alzheimer's disease. Proc. Natl Acad. Sci. USA 99, 10843-10848 (2002).
19. Wojtas, A. M. et al. Loss of clusterin shifts amyloid deposition to the cerebrovasculature via disruption of perivascular drainage pathways. Proc. Natl Acad. Sci. USA 114, E6962-E6971 (2017).

20. Oh, S. B. et al. Clusterin contributes to early stage of Alzheimer's disease pathogenesis. Brain Pathol. 29, 217-231 (2019).

21. Qi, X. M., Wang, C., Chu, X. K., Li, G. \& Ma, J. F. Intraventricular infusion of clusterin ameliorated cognition and pathology in Tg6799 model of Alzheimer's disease. BMC Neurosci. 19, 2 (2018).

22. Fernandez-de-Retana, S. et al. Characterization of ApoJ-reconstituted highdensity lipoprotein (rHDL) nanodisc for the potential treatment of cerebral beta-amyloidosis. Sci. Rep. 7, 14637 (2017).

23. Montoliu-Gaya, L. et al. Differential effects of apoE and apoJ mimetic peptides on the action of an anti-Abeta scFv in 3xTg-AD mice. Biochem. Pharm. 155, 380-392 (2018).

24. Killick, R. et al. Clusterin regulates beta-amyloid toxicity via Dickkopf-1driven induction of the wnt-PCP-JNK pathway. Mol. Psychiatry 19, 88-98 (2014).

25. Robbins, J. P. et al. Clusterin is required for beta-amyloid toxicity in human iPSC-derived neurons. Front Neurosci. 12, 504 (2018).

26. May, P. C. et al. Dynamics of gene expression for a hippocampal glycoprotein elevated in Alzheimer's disease and in response to experimental lesions in rat. Neuron 5, 831-839 (1990)

27. Nilselid, A. M. et al. Clusterin in cerebrospinal fluid: analysis of carbohydrates and quantification of native and glycosylated forms. Neurochem Int. 48, 718-728 (2006).

28. Choi-Miura, N. H. et al. SP-40,40 is a constituent of Alzheimer's amyloid. Acta Neuropathol. 83, 260-264 (1992).

29. McGeer, P. L., Kawamata, T. \& Walker, D. G. Distribution of clusterin in Alzheimer brain tissue. Brain Res. 579, 337-341 (1992).

30. Giannakopoulos, P. et al. Possible neuroprotective role of clusterin in Alzheimer's disease: a quantitative immunocytochemical study. Acta Neuropathol. 95, 387-394 (1998).

31. Itakura, E., Chiba, M., Murata, T. \& Matsuura, A. Heparan sulfate is a clearance receptor for aberrant extracellular proteins. J Cell Biol 219, https:// doi.org/10.1083/jcb.201911126 (2020)

32. Yeh, F. L., Wang, Y., Tom, I., Gonzalez, L. C. \& Sheng, M. TREM2 binds to apolipoproteins, including APOE and CLU/APOJ, and thereby facilitates uptake of amyloid-beta by microglia. Neuron 91, 328-340 (2016).

33. Bell, R. D. et al. Transport pathways for clearance of human Alzheimer's amyloid beta-peptide and apolipoproteins $\mathrm{E}$ and $\mathrm{J}$ in the mouse central nervous system. J. Cereb. Blood Flow. Metab. 27, 909-918 (2007).

34. Thambisetty, M. et al. Association of plasma clusterin concentration with severity, pathology, and progression in Alzheimer disease. Arch. Gen. Psychiatry 67, 739-748 (2010).

35. Schrijvers, E. M., Koudstaal, P. J., Hofman, A. \& Breteler, M. M. Plasma clusterin and the risk of Alzheimer disease. JAMA 305, 1322-1326 (2011)

36. Braak, H., Thal, D. R., Ghebremedhin, E. \& Del Tredici, K. Stages of the pathologic process in Alzheimer disease: age categories from 1 to 100 years. J. Neuropathol. Exp. Neurol. 70, 960-969 (2011).

37. Arriagada, P. V., Growdon, J. H., Hedley-Whyte, E. T. \& Hyman, B. T. Neurofibrillary tangles but not senile plaques parallel duration and severity of Alzheimer's disease. Neurology 42, 631-639 (1992).

38. Nelson, P. T. et al. Correlation of Alzheimer disease neuropathologic changes with cognitive status: a review of the literature. J. Neuropathol. Exp. Neurol. 71, 362-381 (2012)

39. Hsieh, Y. C. et al. Tau-mediated disruption of the spliceosome triggers cryptic RNA splicing and neurodegeneration in Alzheimer's disease. Cell Rep. 29, 301-316 e310 (2019).

40. Wojtas, A. M. et al. Clusterin ameliorates tau pathology in vivo by inhibiting fibril formation. Acta Neuropathol. Commun. 8, 210 (2020)

41. Holmes, B. B. et al. Proteopathic tau seeding predicts tauopathy in vivo. Proc Natl Acad. Sci. USA 111, E4376-E4385 (2014).

42. Barghorn, S. \& Mandelkow, E. Toward a unified scheme for the aggregation of tau into Alzheimer paired helical filaments. Biochemistry 41, 14885-14896 (2002).

43. von Bergen, M., Barghorn, S., Biernat, J., Mandelkow, E. M. \& Mandelkow, E. Tau aggregation is driven by a transition from random coil to beta sheet structure. Biochim. Biophys. Acta 1739, 158-166 (2005).

44. Scheres, S. H., Zhang, W., Falcon, B. \& Goedert, M. Cryo-EM structures of tau filaments. Curr. Opin. Struct. Biol. 64, 17-25 (2020).

45. Goedert, M. et al. Assembly of microtubule-associated protein tau into Alzheimer-like filaments induced by sulphated glycosaminoglycans. Nature 383, 550-553 (1996).

46. Arosio, P., Knowles, T. P. \& Linse, S. On the lag phase in amyloid fibril formation. Phys. Chem. Chem. Phys. 17, 7606-7618 (2015).

47. Mok, S. A. et al. Mapping interactions with the chaperone network reveals factors that protect against tau aggregation. Nat. Struct. Mol. Biol. 25, 384-393 (2018). 
48. Iqbal, K., Liu, F. \& Gong, C. X. Tau and neurodegenerative disease: the story so far. Nat. Rev. Neurol. 12, 15-27 (2016).

49. Goedert, M., Jakes, R. \& Vanmechelen, E. Monoclonal antibody AT8 recognises tau protein phosphorylated at both serine 202 and threonine 205 . Neurosci. Lett. 189, 167-169 (1995).

50. Wischik, C. M. et al. Structural characterization of the core of the paired helical filament of Alzheimer disease. Proc. Natl Acad. Sci. USA 85, 4884-4888 (1988).

51. Dujardin, S. et al. Tau molecular diversity contributes to clinical heterogeneity in Alzheimer's disease. Nat. Med. 26, 1256-1263 (2020).

52. Dujardin, S. \& Hyman, B. T. Tau prion-like propagation: state of the art and current challenges. Adv. Exp. Med. Biol. 1184, 305-325 (2019).

53. Kumar, S. et al. Stages and conformations of the Tau repeat domain during aggregation and its effect on neuronal toxicity. J. Biol. Chem. 289, 20318-20332 (2014).

54. Chen, J. J. et al. Compromised function of the ESCRT pathway promotes endolysosomal escape of tau seeds and propagation of tau aggregation. J. Biol. Chem. 294, 18952-18966 (2019).

55. Falcon, B., Noad, J., McMahon, H., Randow, F. \& Goedert, M. Galectin-8mediated selective autophagy protects against seeded tau aggregation. J. Biol. Chem. 293, 2438-2451 (2018).

56. Rauch, J. N. et al. Tau internalization is regulated by 6-O sulfation on heparan sulfate proteoglycans (HSPGs). Sci. Rep. 8, 6382 (2018).

57. Holmes, B. B. et al. Heparan sulfate proteoglycans mediate internalization and propagation of specific proteopathic seeds. Proc. Natl Acad. Sci. USA 110, E3138-E3147 (2013).

58. Flavin, W. P. et al. Endocytic vesicle rupture is a conserved mechanism of cellular invasion by amyloid proteins. Acta Neuropathol. 134, 629-653 (2017).

59. Yoshimori, T., Yamamoto, A., Moriyama, Y., Futai, M. \& Tashiro, Y. Bafilomycin A1, a specific inhibitor of vacuolar-type $\mathrm{H}(+)$-ATPase, inhibits acidification and protein degradation in lysosomes of cultured cells. J. Biol. Chem. 266, 17707-17712 (1991).

60. Thiele, D. L. \& Lipsky, P. E. Mechanism of L-leucyl-L-leucine methyl estermediated killing of cytotoxic lymphocytes: dependence on a lysosomal thiol protease, dipeptidyl peptidase I, that is enriched in these cells. Proc. Natl Acad. Sci. USA 87, 83-87 (1990).

61. Alavi Naini, S. M. \& Soussi-Yanicostas, N. Heparan sulfate as a therapeutic target in tauopathies: insights from zebrafish. Front Cell Dev. Biol. 6, 163 (2018).

62. Hurley, J. H. ESCRTs are everywhere. EMBO J. 34, 2398-2407 (2015).

63. Thurston, T. L., Wandel, M. P., von Muhlinen, N., Foeglein, A. \& Randow, F. Galectin 8 targets damaged vesicles for autophagy to defend cells against bacterial invasion. Nature 482, 414-418 (2012).

64. Whiten, D. R. et al. Single-molecule characterization of the interactions between extracellular chaperones and toxic alpha-synuclein oligomers. Cell Rep. 23, 3492-3500 (2018)

65. Nachman, E. et al. Disassembly of Tau fibrils by the human Hsp70 disaggregation machinery generates small seeding-competent species. J. Biol. Chem. 295, 9676-9690 (2020).

66. Takeda, S. et al. Seed-competent high-molecular-weight tau species accumulates in the cerebrospinal fluid of Alzheimer's disease mouse model and human patients. Ann. Neurol. 80, 355-367 (2016).

67. Wang, C. et al. Selective removal of astrocytic APOE4 strongly protects against tau-mediated neurodegeneration and decreases synaptic phagocytosis by microglia. Neuron, https://doi.org/10.1016/j.neuron.2021.03.024 (2021).

68. Litvinchuk, A. et al. Apolipoprotein E4 reduction with antisense oligonucleotides decreases neurodegeneration in a tauopathy model. Ann. Neurol. 89, 952-966 (2021).

69. Shi, Y. et al. ApoE4 markedly exacerbates tau-mediated neurodegeneration in a mouse model of tauopathy. Nature 549, 523-527 (2017).

70. Zhao, N. et al. APOE epsilon2 is associated with increased tau pathology in primary tauopathy. Nat. Commun. 9, 4388 (2018).

71. Kwon, M. J. et al. Deficiency of clusterin exacerbates high-fat diet-induced insulin resistance in male mice. Endocrinology 155, 2089-2101 (2014).

72. DeMattos, R. B. et al. ApoE and clusterin cooperatively suppress Abeta levels and deposition: evidence that ApoE regulates extracellular Abeta metabolism in vivo. Neuron 41, 193-202 (2004).

73. Stone, D. J., Rozovsky, I., Morgan, T. E., Anderson, C. P. \& Finch, C. E. Increased synaptic sprouting in response to estrogen via an apolipoprotein Edependent mechanism: implications for Alzheimer's disease. J. Neurosci. 18, 3180-3185 (1998).

74. An, N. et al. Synergistic effects of APOE and CLU may increase the risk of Alzheimer's disease: acceleration of atrophy in the volumes and shapes of the hippocampus and amygdala. J. Alzheimers Dis. 80, 1311-1327 (2021).

75. Lenzi, C. et al. The down-regulation of clusterin expression enhances the alphasynuclein aggregation process. Int J Mol Sci 21, https://doi.org/10.3390/ ijms21197181 (2020).
76. Filippini, A. et al. Extracellular clusterin limits the uptake of alpha-synuclein fibrils by murine and human astrocytes. Glia, https://doi.org/10.1002/ glia.23920 (2020).

77. Vranova, H. P. et al. CSF markers of neurodegeneration in Parkinson's disease. J. Neural Transm. 117, 1177-1181 (2010).

78. Buchman, A. S. et al. Progressive parkinsonism in older adults is related to the burden of mixed brain pathologies. Neurology 92, E1821-E1830 (2019).

79. Irwin, D. J., Lee, V. M. Y. \& Trojanowski, J. Q. Parkinson's disease dementia: convergence of alpha-synuclein, tau and amyloid-beta pathologies. Nat. Rev. Neurosci. 14, 626-636 (2013).

80. Goedhart, J. et al. Structure-guided evolution of cyan fluorescent proteins towards a quantum yield of 93\%. Nat. Commun. 3, 751 (2012).

81. Hoover, B. R. et al. Tau mislocalization to dendritic spines mediates synaptic dysfunction independently of neurodegeneration. Neuron 68, 1067-1081 (2010).

82. Catanzariti, A. M., Soboleva, T. A., Jans, D. A., Board, P. G. \& Baker, R. T. An efficient system for high-level expression and easy purification of authentic recombinant proteins. Protein Sci. 13, 1331-1339 (2004).

83. Hedgepeth, C. M. et al. Activation of the Wnt signaling pathway: a molecular mechanism for lithium action. Dev. Biol. 185, 82-91 (1997).

84. Rospigliosi, C. C. et al. E46K Parkinson's-linked mutation enhances Cterminal-to-N-terminal contacts in alpha-synuclein. J. Mol. Biol. 388, 1022-1032 (2009).

85. Furlong, R. A., Narain, Y., Rankin, J., Wyttenbach, A. \& Rubinsztein, D. C. Alpha-synuclein overexpression promotes aggregation of mutant huntingtin. Biochem J. 346, 577-581 (2000).

86. Durocher, Y., Perret, S. \& Kamen, A. High-level and high-throughput recombinant protein production by transient transfection of suspensiongrowing human 293-EBNA1 cells. Nucleic Acids Res. 30, E9 (2002).

87. Li, Z., Michael, I. P., Zhou, D., Nagy, A. \& Rini, J. M. Simple piggyBac transposon-based mammalian cell expression system for inducible protein production. Proc. Natl Acad. Sci. USA 110, 5004-5009 (2013).

88. Trinkaus, V. A. et al. In situ architecture of neuronal alpha-Synuclein inclusions. Nat. Commun. 12, 2110 (2021).

89. Volpicelli-Daley, L. A., Luk, K. C. \& Lee, V. M. Addition of exogenous alphasynuclein preformed fibrils to primary neuronal cultures to seed recruitment of endogenous alpha-synuclein to Lewy body and Lewy neurite-like aggregates. Nat. Protoc. 9, 2135-2146 (2014).

90. Imamoglu, R., Balchin, D., Hayer-Hartl, M. \& Hartl, F. U. Bacterial Hsp70 resolves misfolded states and accelerates productive folding of a multi-domain protein. Nat. Commun. 11, 365 (2020).

91. Gupta, A. J., Haldar, S., Milicic, G., Hartl, F. U. \& Hayer-Hartl, M. Active cage mechanism of chaperonin-assisted protein folding demonstrated at singlemolecule level. J. Mol. Biol. 426, 2739-2754 (2014).

92. Wilkins, D. K. et al. Hydrodynamic radii of native and denatured proteins measured by pulse field gradient NMR techniques. Biochemistry $\mathbf{3 8}$, 16424-16431 (1999).

93. Banning, C. et al. A flow cytometry-based FRET assay to identify and analyse protein-protein interactions in living cells. PLoS ONE 5, e9344 (2010).

\section{Acknowledgements}

We thank Marc Diamond for the N1-TauRD (P301L/V337M)-EYFP plasmid; Michael Gropp for the pHUE-TauRD (P301L/V337M) plasmid; Saurabh Gautam for FLTau protein; Itika Saha for sharing aggregate seeding protocols; Prof. Wolfgang Baumeister and his department for microscope support; Martin Spitaler, Markus Oster, and Giovanni Cardone from the Max Planck Institute of Biochemistry (MPIB) imaging facility for support with flow cytometry, imaging and image processing; Sabine Suppmann and Judith Scholz from the MPIB Biochemistry core facility for producing recombinant Clusterin; the MPIB cryo-EM facility for EM support; David Balchin for helpful discussion; Gopal Jayaraj for commenting on the manuscript and helpful discussion. The research leading to these results has received funding from the European Commission under Grant FP7 GA ERC- 2012-SyG_318987-ToPAG, the Deutsche Forschungsgemeinschaft (DFG, German Research Foundation) under Germany's Excellence Strategy within the framework of the Munich Cluster for Systems Neurology (EXC 2145 SyNergy-ID 390857198) and by the joint efforts of The Michael J. Fox Foundation for Parkinson's Research (MJFF) and the Aligning Science Across Parkinson's (ASAP) initiative. MJFF administers the grant ASAP-000282 on behalf of ASAP and itself. For the purpose of open access, the authors have applied a CC-BY public copyright license to the Author Accepted Manuscript version arising from this submission.

\section{Author contributions}

F.U.H. conceived the project. P.Y. designed, performed, and analyzed most of the research. V.T. conducted experiments with $\alpha$-Syn. I.R. performed experiments with mouse primary neurons. R.I. conducted and analyzed dcFCCS experiments. T.S. optimized and purified recombinant Clusterin. H.W. performed Tau negative stain electron 
microscopy. I.D. supervised experiments with primary neurons. M.S.H. initially cosupervised the project and commented on the manuscript. A.B. co-supervised the project and contributed to experimental design. P.Y., A.B., and F.U.H. wrote the manuscript with input from the other authors.

\section{Funding}

Open Access funding enabled and organized by Projekt DEAL.

\section{Competing interests}

The authors declare no competing interests.

\section{Additional information}

Supplementary information The online version contains supplementary material available at https://doi.org/10.1038/s41467-021-25060-1.

Correspondence and requests for materials should be addressed to F.U.H.

Peer review information Nature Communications thanks Lukasz Joachimiak and the other, anonymous, reviewer(s) for their contribution to the peer review of this work.

Reprints and permission information is available at http://www.nature.com/reprints

Publisher's note Springer Nature remains neutral with regard to jurisdictional claims in published maps and institutional affiliations.


appropriate credit to the original author(s) and the source, provide a link to the Creative Commons license, and indicate if changes were made. The images or other third party material in this article are included in the article's Creative Commons license, unless indicated otherwise in a credit line to the material. If material is not included in the article's Creative Commons license and your intended use is not permitted by statutory regulation or exceeds the permitted use, you will need to obtain permission directly from the copyright holder. To view a copy of this license, visit http://creativecommons.org/ licenses/by/4.0/.

(C) The Author(s) 2021 